(C) 2010 International Press

Adv. Theor. Math. Phys. 14 (2010) 609-641

\title{
Instantons on the Taub-NUT space
}

\author{
Sergey A. Cherkis \\ School of Mathematics and Hamilton Mathematics Institute, \\ Trinity College Dublin, Ireland \\ cherkis@maths.tcd.ie
}

\begin{abstract}
We present a construction of self-dual Yang-Mills connections on the Taub-NUT space. We illustrate it by finding explicit expressions for all $\mathrm{SU}(2)$ instantons of instanton number one and generic monodromy at infinity.
\end{abstract}

\section{Contents}

1 Introduction

1.1 Background

1.2 Instanton number and monopole charges

2 Ingredients

2.1 Arrows and limbs

2.2 The string

3 The Taub-NUT as a hyperkähler quotient 
3.2 The family of connections $\quad 618$

$\begin{array}{lll}3.3 & \text { A basis of self-dual two forms } & 621\end{array}$

4 Instanton data $\quad 622$

5 The Nahm transform $\quad 623$

5.1 The Weyl operator $\quad 623$

$\begin{array}{lll}5.2 & \text { The connection } & 625\end{array}$

6 The ADHM limit $\quad 626$

7 Proof of self-duality $\quad 627$

8 Instantons for the $U(n)$ gauge group 628

9 The geometric meaning of the Nahm transform for $\begin{array}{ll}\text { curved manifolds } & 629\end{array}$

10 Example of one instanton 631

10.1 Solving the Weyl equation 632

10.2 Normalization $\quad 634$

$\begin{array}{lll}10.3 & \text { Connection } & 635\end{array}$

11 Conclusions $\quad 636$

$\begin{array}{ll}\text { Acknowledgments } & 637\end{array}$

$\begin{array}{ll}\text { Appendix A. } & 637\end{array}$

A.1 Metric conventions and moment maps 637

A.2 Vanishing moment map conditions 638

A.3 The Weyl equation 638

A.4 Solution of the Weyl equation 639

$\begin{array}{ll}\text { References } & 639\end{array}$ 


\section{Introduction}

An instanton on a Taub-NUT space is a connection, given by a $u(n)$-valued one form i $A$, on an $n$-dimensional Hermitian bundle $\mathcal{E}$ over the Taub-NUT space with the curvature two form $F=d A-\mathrm{i} A \wedge A$ satisfying the selfduality condition

$$
F=* F
$$

Here $*$ denotes the Hodge star operator taking a two form to its dual. We require the connection $A$ to have finite action $S=\int \operatorname{tr} F \wedge * F$.

Everywhere outside one point 0 the Taub-NUT space itself can be thought of as being fibered by a circle $S^{1}$ over a base $\mathbb{R}^{3} \backslash 0$. Choosing $\tau \sim \tau+4 \pi$ to be the periodic coordinate on the $S^{1}$ fiber and $\vec{x}=\left(x_{1}, x_{2}, x_{3}\right)$, with $x_{1}, x_{2}$, and $x_{3}$ coordinates on $\mathbb{R}^{3}$, the Taub-NUT metric ${ }^{1}$ is

$$
d s^{2}=\frac{1}{4}\left(\left(l+\frac{1}{|\vec{x}|}\right) d \vec{x}^{2}+\frac{1}{\left(l+\frac{1}{|\vec{x}|}\right)}(d \tau+\vec{\omega} \cdot d \vec{x})^{2}\right)
$$

where 1 is some fixed parameter determining the asymptotic size of the $S^{1}$ and $\frac{\partial}{\partial x_{i}} \frac{1}{|\vec{x}|}=\epsilon_{i j k} \frac{\partial}{\partial x_{j}} \omega_{k}$. This metric degenerates to a flat metric on $\mathbb{R}^{4}$ as $l \rightarrow 0$. Its noncompact cycle $\mathcal{C}:\left\{(\tau, \vec{x}) \mid x_{1}=x_{2}=0, x_{3} \geq 0\right\}$ becomes a plane in this limit.

The Taub-NUT space is equipped with a natural line bundle with a connection $a=\frac{1}{2 V}(d \tau+\omega)$. This connection has a self-dual curvature $d a$. As a matter of fact, it has a one parameter family of such line bundles with the following Abelian connections:

$$
a_{s}=s a=\frac{s}{2} \quad \frac{d \tau+\omega}{V}
$$

parameterized by $s \in[-l / 2, l / 2]$. These connections are Abelian instantons, as their curvature is self-dual in the orientation $\left(\tau, x_{1}, x_{2}, x_{3}\right)$ and has a finite

\footnotetext{
${ }^{1}$ The factor of $\frac{1}{4}$ in the metric is chosen for future convenience and the apparent singularity at the origin of $\mathbb{R}^{3}$ is merely a coordinate singularity.
} 
action. Note, that the relation between the left and right ends of this interval is given by tensoring with a line bundle $\mathcal{L}_{l}$, which is trivial since

$$
\int_{C} d\left(a_{l / 2}-a_{-l / 2}\right)=2 \pi
$$

\subsection{Background}

There has been a lot of work exploring instantons in various backgrounds. The Atiyah-Drinfeld-Hitchin-Manin (ADHM) original construction [1] provides all instantons on $\mathbb{R}^{4}$. Nahm modified this construction in $[2,3]$ to provide calorons, i.e., instantons on $\mathbb{R}^{3} \times S^{1}$. Orbifolding the ADHM construction Kronheimer and Nakajima [4] obtained instantons on Asymptotically Locally Euclidean (ALE) spaces. In [5] Nekrasov and Schwarz modified the ADHM construction to construct instantons on noncommutative $\mathbb{R}^{4}$. All of these constructions have string theory interpretations $[6-8]$ and emerge from the sigma model analysis of appropriate D-brane configurations.

Based on these general constructions some explicit solutions at a general position were obtained in $[9,10]$ for a caloron and in [11] for instantons on certain ALE spaces.

We would like to point out that in all these cases the underlying space is flat, or it has a useful flat limit. Here, we aim to find a general construction for generic ${ }^{2}$ instantons on an essentially curved space. In particular, building on the bow formalism introduced in [16] to study the moduli spaces of instantons on the Taub-NUT space, we find expressions for the instanton connection. As an illustration of our construction we find the explicit general solution for a single instanton on a Taub-NUT space.

\subsection{Instanton number and monopole charges}

A generic self-dual $U(n)$ configuration on the Taub-NUT space possesses two types of topological charges: an instanton number $k_{0}$ and $n$ monopole charges $m_{1}, m_{2}, \ldots, m_{n}$. The instanton number as well as the monopole charges are given by integers. A detailed discussion of various charges of instantons on muti-Taub-NUT spaces and their relation with the corresponding brane configurations appeared recently in [17]. Here we define charges in a somewhat different fashion.

\footnotetext{
${ }^{2}$ Some special instanton solutions on the Taub-NUT space were obtained in [12-15].
} 
For any given $\vec{x} \in \mathbb{R}$ consider the monodromy $W(\vec{x}, \tau) \in U(n)$ satisfying $\left(\partial_{\tau}-\mathrm{i} A_{\tau}\right) W(\vec{x}, \tau)=0$ and $W(\vec{x}, 0)=1$, so that the monodromy around the circle $S_{\vec{x}}^{1}$ is $W(\vec{x}, 4 \pi)$. The finite action condition implies that the conjugacy class of $\lim _{|\vec{x}| \rightarrow \infty} W(\vec{x}, 4 \pi)$ is well defined and does not depend on the direction in which we approach infinity. We write the eigenvalues of $\lim _{|\vec{x}| \rightarrow \infty} W(\vec{x}, 4 \pi)$ as

$$
\exp \left(\frac{2 \pi \mathrm{i} \lambda_{1}}{l}\right), \exp \left(\frac{2 \pi \mathrm{i} \lambda_{2}}{l}\right), \ldots, \exp \left(\frac{2 \pi \mathrm{i} \lambda_{n}}{l}\right) .
$$

Here we restrict our attention to the so-called "maximal symmetry breaking" case presuming all $\lambda_{j}$ are distinct and ordered: $-\frac{l}{2}<\lambda_{1}<\lambda_{2}<\cdots<$ $\lambda_{n}<\frac{l}{2}$.

Consider a sphere $S_{R}^{2}=\{\vec{x}|| \vec{x} \mid=R\} \in \mathbb{R}^{3}$ of large radius $R$. Any point on this sphere determines a $\tau$-circle in the Taub-NUT space, so that the union of all these circles is a squashed three sphere $S_{R}^{3}$. Thus, $S_{R}^{3}$ is fibered by circles over the $S_{R}^{2}$ and, for a Taub-NUT space, this fibration is the Hopf fibration $S^{1} \rightarrow S_{R}^{3} \rightarrow S_{R}^{2}$. Since the total action is finite there is a gauge transformation on $S_{R}^{3}$ such that for large radius $R$ the connection $A$ restricted to $S_{R}^{3}$ approaches one with $\tau$-independent components. Let us write this connection with $\tau$-independent components in the form $A=\hat{A}-\hat{\Phi} \frac{d \tau+\omega}{V}$. Then, the self-duality condition for $A$ is equivalent [18] to the Bogomolny equation

$$
\hat{F}=*_{3} D_{\hat{A}} \hat{\Phi}
$$

for $(\hat{A}, \hat{\Phi})$. Here $*_{3}$ is the three-dimensional Hodge star operator for the flat metric $d x_{1}^{2}+d x_{2}^{2}+d x_{3}^{2}$ and $\hat{F}$ is the curvature form of $\hat{A}$. The asymptotic eigenvalues of $\hat{\Phi}$ are determined by the eigenvalues of the monodromy operator $W(\vec{x}, 4 \pi)$. Moreover, since the asymptotic behavior of $\hat{\Phi}$ eigenvalues is the same as for a Bogomol'nyi-Prasad-Sommerfield (BPS) monopole, the eigenvalues of $\hat{\Phi}$ are

$$
\lambda_{1}+\frac{j_{1}}{x}+O\left(x^{-2}\right), \quad \lambda_{2}+\frac{j_{2}}{x}+O\left(x^{-2}\right), \ldots, \quad \lambda_{n}+\frac{j_{n}}{x}+O\left(x^{-2}\right),
$$

with $j_{1}, j_{2}, \ldots, j_{n}$ integers.

Let us describe this construction in different terms, making clear that $j$ 's are indeed integers. Considering the eigenspaces of the monodromy operator $W(\vec{x}, \tau+4 \pi) W^{-1}(\vec{x}, \tau)$ we split the bundle $\left.\mathcal{E}\right|_{S_{R}^{3}}$ into $k$ eigenline bundles $\left.\mathcal{E}\right|_{S_{R}^{3}}=\mathcal{L}_{\lambda_{1}} \oplus \mathcal{L}_{\lambda_{2}} \oplus \ldots \oplus \mathcal{L}_{\lambda_{n}}$. Since each eigenvalue $\lambda$ is independent of the base, each of these line bundles can be trivialized on all $S^{1}$ Hopf fibers 
simultaneously. Thus, we have a well-defined pushdown line bundles over the base of the Hopf fibration $S_{R}^{2}$. Chern classes of these are $j_{1}, j_{2}, \ldots, j_{n}$. We now use these integers to define the monopole charges of the configuration.

Let $M=\min \left(j_{1}, j_{1}+j_{2}, \ldots, j_{1}+j_{2}+\cdots+j_{n}\right)$. The monopole charges of an instanton on a Taub-NUT are defined as

$$
\left(m_{1}, m_{2}, \ldots, m_{n}\right)=\left(j_{1}-M, j_{1}+j_{2}-M, \ldots, j_{1}+j_{2}+\cdots+j_{n}-M\right) .
$$

Note, that from the way they are defined, one of these charges, say $m_{p}$, must vanish. Nevertheless, we keep it among the charges and its position $p$ is significant as will be clear momentarily.

Intuitively, since the total action is finite, the asymptotic connection can be put into a form independent of the $\tau$ coordinate. Then, asymptotically, it can be reduced to a monopole on the base $\mathbb{R}^{3}[18]$. It is the charges of this monopole that we defined above.

The instanton number is less straightforward to define. One can write an explicit expression given by the Chern number minus the contributions of the monopole charges. To make clear that it is integer, we define it here as an index of the Weyl operator for the connection $A+\frac{1}{2}\left(\lambda_{p}+\lambda_{p+1}\right) a$ :

$$
k_{0}=\operatorname{Ind} D_{A+\frac{1}{2}\left(\lambda_{p}+\lambda_{p+1}\right) a} .
$$

Thus a general $U(n)$ instanton on a Taub-NUT has an instanton number $k_{0}$ and monopole charges $\left(m_{1}, m_{2}, \ldots, m_{n}\right)$.

Kronheimer [18] demonstrated equivalence of the "pure monopole" case, i.e., the case with $k_{0}=0$, to singular monopoles studied in $[19,20]$. In particular, explicit solutions for $k_{0}=0$ and $m=1$ (that is $(1,0)$ monopole charges) are equivalent to singular monopole solutions presented in [21,22]. In this paper we focus our attention on the pure instanton case of vanishing monopole charges, and obtain explicit solutions with $k_{0}=1$, i.e., a single $\mathrm{SU}(2)$ instanton on the Taub-NUT space with no monopole charge. The explicit metric on the moduli space of such solutions was found in [16].

\section{Ingredients}

The data specifying an instanton on a Taub-NUT space will be encoded in terms of a bow diagram. There are two basic ingredients in our construction: arrows and strings. 


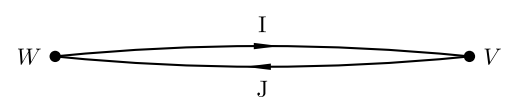

(a) Linear maps (arrows and limbs). (b) Nahm Data (string).

Figure 1: Components of bow diagrams.

\subsection{Arrows and limbs}

Figure 1(a) represents a pair of complex vector spaces $V=\mathbb{C}^{v}$ and $W=\mathbb{C}^{w}$ with maps $J: V \rightarrow W$ and $I: W \rightarrow V$. The linear space formed by the pair of maps $(I, J)$ has a natural hyperkähler structure, which is respected by the action of $U(v)$ and $U(w)$. The hyperkähler moment map of the $U(v)$ action $g_{v}:(I, J) \mapsto\left(g_{v}^{-1} I, J g_{v}\right)$ is given by

$$
\mu_{V}^{\mathbb{C}}=\mu_{V}^{1}+\mathrm{i} \mu_{V}^{2}=I J, \quad \mu_{V}^{\mathbb{R}}=\mu_{V}^{3}=\frac{1}{2}\left(J^{\dagger} J-I I^{\dagger}\right),
$$

while for the $U(w)$ action $g_{w}:(I, J) \mapsto\left(I g_{w}, g_{w}^{-1} J\right)$ the moment map is

$$
\mu_{W}^{\mathbb{C}}=\mu_{W}^{1}+\mathrm{i} \mu_{W}^{2}=-J I, \quad \mu_{W}^{\mathbb{R}}=\mu_{W}^{3}=\frac{1}{2}\left(I^{\dagger} I-J J^{\dagger}\right) .
$$

It is convenient to assemble the pair $(I, J)$ into

$$
Q=\left(\begin{array}{c}
J^{\dagger} \\
I
\end{array}\right) \quad \text { and } \quad \bigcirc=\left(\begin{array}{c}
c I^{\dagger} \\
-J
\end{array}\right)
$$

(pronounced "kyu" and "yuk") so that $Q: W \rightarrow S \otimes V$ and $\bigcirc: V \rightarrow S \otimes W$ with the three complex structures $e_{j}=-i \sigma_{j}$ acting on $Q$ 's. $S \approx \mathbb{C}^{2}$ is a two dimensional space of spinors providing the representation of quaternions, with the quaternionic units $e_{j}=i \sigma_{j}$, i.e.,

$$
e_{1}=-\mathrm{i}\left(\begin{array}{ll}
0 & 1 \\
1 & 0
\end{array}\right), \quad e_{2}=-\mathrm{i}\left(\begin{array}{cc}
0 & -i \\
i & 0
\end{array}\right), \quad e_{3}=-\mathrm{i}\left(\begin{array}{cc}
1 & 0 \\
0 & -1
\end{array}\right)
$$

The natural metric on the linear space of all pairs of maps is

$$
d s^{2}=\operatorname{tr}_{W} d Q^{\dagger} d Q=\operatorname{tr}_{W}\left(d J d J^{\dagger}+d I^{\dagger} d I\right)
$$

The tree symplectic forms

$$
\omega_{j} \equiv g\left(\cdot, e_{j}\right)=\frac{1}{2} \operatorname{tr}_{W}\left(d Q^{\dagger} \wedge e_{j} d Q\right),
$$


can be combined into $\downarrow \equiv \omega_{j} \sigma_{j}=\mathrm{iVec} \operatorname{tr}_{V} d Q \wedge d Q^{\dagger}$. Here we introduce a "vector operation" Vec defined by

$$
\operatorname{Vec}\left(1_{2 \times 2} \otimes M^{0}+\sigma_{j} \otimes M^{j}\right)=\sigma_{j} \otimes M^{j} .
$$

Since $-\mathrm{i} \sigma_{j}$ represent the quaternionic imaginary units, this operation basically amounts to taking the imaginary part of a quaternion.

With this notation the moment maps are

$$
\lambda_{\iota_{V}}=\mu_{V}^{i} \sigma_{i}=\operatorname{Vec}\left(Q Q^{\dagger}\right) \text { and } \lambda_{l_{W}}=\mu_{W}^{i} \sigma_{i}=\operatorname{Vec}\left(O O^{\dagger}\right) .
$$

\section{$2.2 \quad$ The string}

Figure 1(b) represents an interval $\mathcal{I}$ parameterized by $s$ with a bundle $E \rightarrow \mathcal{I}$ endowed with a Hermitian structure, a connection $D_{s}=d / d s+\mathrm{i} T_{0}$, and a triplet $\vec{T}=\left(T_{1}, T_{2}, T_{3}\right)$ of endomorphisms of $E$. In other words for a given trivialization of $E$, we have a quadruplet of Hermitian matrix valued functions $\left(T_{0}(s), T_{1}(s), T_{2}(s), T_{3}(s)\right)$. These also form a linear space with a natural flat metric $d s^{2}=\int \operatorname{tr}_{E}\left(d T_{0}^{2}+d T_{1}^{2}+d T_{2}^{2}+d T_{3}^{2}\right)$ and a hyperkähler structure all invariant with respect to the following gauge group action:

$$
g(s):\left(\begin{array}{c}
T_{0}(s) \\
T_{1}(s) \\
T_{2}(s) \\
T_{3}(s)
\end{array}\right) \mapsto\left(\begin{array}{c}
g^{-1} T_{0} g-\mathrm{i} g^{-1} \frac{d}{d s} g \\
g^{-1} T_{1} g \\
g^{-1} T_{2} g \\
g^{-1} T_{3} g
\end{array}\right) .
$$

The corresponding moment maps are

$$
\begin{aligned}
\mu^{1} & =\frac{d}{d s} T_{1}+\mathrm{i}\left[T_{0}, T_{1}\right]+\mathrm{i}\left[T_{2}, T_{3}\right], \\
\mu^{2} & =\frac{d}{d s} T_{2}+\mathrm{i}\left[T_{0}, T_{2}\right]+\mathrm{i}\left[T_{3}, T_{1}\right], \\
\mu^{3} & =\frac{d}{d s} T_{3}+\mathrm{i}\left[T_{0}, T_{3}\right]+\mathrm{i}\left[T_{1}, T_{2}\right] .
\end{aligned}
$$

It is convenient to introduce $\mathbb{F}=\sigma_{1} \otimes T_{1}+\sigma_{2} \otimes T_{2}+\sigma_{3} \otimes T_{3}$ so that the moment map $\lambda<=\left[\frac{d}{d s}+\mathrm{i} T_{0}, 7\right]+\operatorname{Vec} T F$.

Assembling the Nahm data into a quaternion $T=T_{0}+T_{j} \otimes e_{j}=T_{0}-$ i 7 we write the above metric on the linear space of all the Nahm data in the form

$$
d s^{2}=\frac{1}{2} \int \operatorname{tr}_{S} \operatorname{tr}_{E} \delta T^{\dagger} \delta T d s .
$$


The three symplectic forms $\omega_{j}=g\left(\cdot, e_{j} \cdot\right)$ are encoded in

$$
\downarrow=\frac{\mathrm{i}}{2} \int \operatorname{tr}_{E} \delta T \wedge \delta T^{\dagger} d s
$$

Note that the moment maps can be written in terms of the Weyl operator $D=-D_{s}+\mp$ and its conjugate $D^{\dagger}=D_{s}+\mp$ as

$$
\lambda_{c}=\operatorname{Vec}\left(D_{s}+\mathbb{F}\right)\left(-D_{s}+\mp\right)
$$

\section{The Taub-NUT as a hyperkähler quotient}

This section contains a description of the Taub-NUT space using the ingredients we have defined in the previous section. This description will naturally lead us to a family of self-dual harmonic forms ${ }^{3}$ which are essential for the instanton construction that follows. Our exposition in this section is close to that of Gibbons and Rychenkova [23]. Just as for the construction [4] of instantons on ALE spaces it was essential to know the realization of the underlying ALE space as a hyperkähler quotient of linear spaces [24], this section contains the realization of the Taub-NUT space as a hyperkähler quotient setting the groundwork for the construction of instantons on it.

\subsection{Taub-NUT bow data}

The bow diagram in figure 2 represents Nahm data of rank 1 associated with a Hermitian line bundle $e \rightarrow \mathcal{I}$ on an interval $[-l / 2, l / 2]$ of length $l$, as well as maps $b_{10}$ and $b_{01}$ between the one-dimensional complex vector spaces $e_{0}=\left.e\right|_{s=-l / 2}$ and $e_{1}=\left.e\right|_{s=l / 2}$ at the ends of the interval. A gauge transformation $h(s)$ acts on these data as follows:

$$
\left(\begin{array}{c}
t_{0} \\
t_{j} \\
b_{01} \\
b_{10}
\end{array}\right) \mapsto\left(\begin{array}{c}
h^{-1} t_{0} h+\mathrm{i} h^{-1} \frac{d}{d s} h \\
h^{-1} t_{j} h \\
h^{-1}\left(-\frac{l}{2}\right) b_{01} h\left(\frac{l}{2}\right) \\
h^{-1}\left(\frac{l}{2}\right) b_{10} h\left(-\frac{l}{2}\right)
\end{array}\right)
$$

\footnotetext{
${ }^{3} \mathrm{~A}$ description of these in terms of the hyperkähler reduction recently appeared in [17].
} 


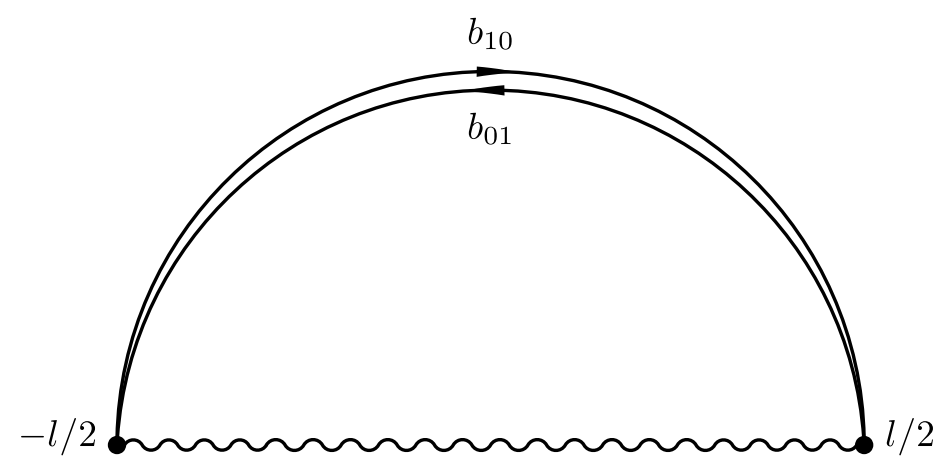

Figure 2: A Taub-NUT bow diagram.

Introducing $\mathbf{t}=t_{1}+\mathrm{i} t_{2}$ and $\mathbf{D}=d / d s-\mathrm{i} t_{0}-t_{3}$ the vanishing of moment maps can be written in complex notation as

$$
\begin{aligned}
& {[\mathbf{D}, \mathbf{t}]-\delta\left(s+\frac{l}{2}\right) b_{01} b_{10}+\delta\left(s-\frac{l}{2}\right) b_{10} b_{01}=0} \\
& {\left[\mathbf{D}^{\dagger}, \mathbf{D}\right]+\left[\mathbf{t}^{\dagger}, \mathbf{t}\right]+\delta\left(s+\frac{l}{2}\right)\left(b_{10}^{\dagger} b_{10}-b_{01} b_{01}^{\dagger}\right)+\delta\left(s-\frac{l}{2}\right)\left(b_{01}^{\dagger} b_{01}-b_{10} b_{10}^{\dagger}\right)=0}
\end{aligned}
$$

Let us distinguish some point $s_{0}$ on the Nahm interval. Say this point divides this interval into two intervals of lengths $l_{L}$ and $l_{R}$, i.e., $l_{L}+l_{R}=l$ and at this distinguished point $s=s_{0}=l_{L}-l / 2=l / 2-l_{R}$. Let us assume $s_{0}>0$. We shall perform the hyperkähler quotient step-by-step, so that the last step is the quotient with respect to the $U(1)$ at the distinguished point ${ }^{4}$ $s_{0}$. This will allow us to associate with any point $s_{0}$ a natural line bundle over the Taub-NUT space and its natural connection corresponding to this $U(1)$.

\subsection{The family of connections}

First we perform hyperkähler reduction on each open interval separately. The intervals are of lengths $l_{L}$ and $l_{R}$. Since the computations are identical, we focus on the interval of length $l_{R}$ to the right of $s_{0}$. As the Nahm data is Abelian, the vanishing of the moment maps implies $d t_{j} / d s=0$, thus, the vector $\vec{t}=\left(t_{1}, t_{2}, t_{3}\right)$ is constant. The connection $t_{0}$ can be made constant using gauge transformations that are trivial at the ends of the interval. There is a large gauge transformation $g=\exp \left(2 \pi \mathrm{i}\left(s-s_{0}\right) / l_{R}\right)$ satisfying $g\left(s_{0}\right)=$ $g(l / 2)=1$. This gauge transformation takes $t_{0 R}$ to $t_{0 R}+2 \pi / l_{R}$. Thus, the result of this hyperkähler reduction is $\mathbb{R}^{3} \times S^{1}$ with coordinates $t_{1 R}, t_{2 R}, t_{3 R}$

\footnotetext{
${ }^{4}$ To be exact, this $U(1)$ is the quotient of the group of all gauge transformation on the interval by the subgroup formed by the gauge transformation that equal to identity at $s_{0}$.
} 
and $t_{0 R} \sim t_{0 R}+2 \pi / l_{R}$ and the metric

$$
d s^{2}=\int_{s_{0}}^{l}\left(d t_{0 R}^{2}+d \vec{t}_{R}^{2}\right) d s=l_{R}\left(d t_{0 R}^{2}+d \vec{t}_{R}^{2}\right) .
$$

The resulting metric on the Nahm data on the left interval is given by the same expression with $l_{R}$ replaced by $l_{L}$.

Now we perform the hyperkähler reduction with respect to the $U(1)$ at $s=l / 2$, which can be realized by $h=\exp \left(\mathrm{i} \phi \frac{s-s_{0}}{l_{R}}\right)$. Exploiting the fact that all the data are Abelian, we assemble the linear data $\left(b_{01}, b_{10}\right)$ into a quaternion

$$
q=q^{0}+q^{i} e_{i}=\left(b_{-}, b_{+}\right)=\left(\begin{array}{cc}
\bar{b}_{01} & \bar{b}_{10} \\
-b_{10} & b_{01}
\end{array}\right) .
$$

Here $b_{-}$and $b_{+}$play the roles of $\bigcirc$ and $Q$. The natural metric is

$$
d s^{2}=\frac{1}{2} \operatorname{tr}_{S} d q^{\dagger} d q=d b_{-}^{\dagger} d b_{-}=d b_{+}^{\dagger} d b_{+},
$$

and the resulting symplectic forms are given by $\downarrow=\mathrm{iVec} d q \wedge d q^{\dagger}$. A gauge transformation $h(s)$ with $h(-l / 2)=\exp \left(\mathrm{i} \phi_{L}\right)$ and $h(l / 2)=\exp \left(\mathrm{i} \phi_{R}\right)$ sends $q$ to $q \exp \left(e_{3}\left(\phi_{R}-\phi_{L}\right)\right)$. The resulting moment maps are $\lambda_{L}{ }_{L}=-\frac{1}{2} q \sigma_{3} q^{\dagger}$ and $\wedge_{R}=\frac{1}{2} q \sigma_{3} q^{\dagger}$.

The rightmost $U(1)$ acts as

$$
\exp \left(\mathrm{i} \phi \frac{s-s_{0}}{l_{R}}\right):\left(q, t_{0 R}, \vec{t}_{R}\right) \mapsto\left(q e^{e_{3} \phi}, t_{0 R}-\phi / l_{R}, \vec{t}_{R}\right),
$$

with the moment map $\mu_{1} e_{1}+\mu_{2} e_{2}+\mu_{3} e_{3}=\frac{1}{2} q e_{3} \bar{q}=t_{1} e_{1}+t_{2} e_{2}+t_{3} e_{3}$. Let $q=a \mathrm{e}^{e_{3} \psi / 2}$ where $a$ is a pure imaginary quaternion, and let $\vec{x}=\left(x_{1}, x_{2}, x_{3}\right)$ be such that $x_{1} e_{1}+x_{2} e_{2}+x_{3} e_{3}=q e_{3} \bar{q}$. The periodic coordinate $\psi \sim \psi+4 \pi$ and the components of $\vec{x}$ provide new coordinates on $\mathbb{R}^{4}$. Then the flat metric on the set of octuplets $\left(t_{0 R}, \vec{t}_{0 R}, b_{01}, b_{10}\right)$ is

$$
\begin{aligned}
d s^{2} & =\frac{1}{2} \operatorname{tr}_{S} d q^{\dagger} d q+l_{R}\left(d t_{0 R}^{2}+d \vec{t}_{R}^{2}\right) \\
& =\frac{1}{4}\left(\frac{1}{|\vec{x}|} d \vec{x}^{2}+|\vec{x}|(d \psi+\omega)^{2}\right)+l_{R}\left(d t_{0 R}^{2}+d \vec{t}_{R}^{2}\right),
\end{aligned}
$$


where

$\mathrm{i}|\vec{x}|(\omega+d \psi)=\frac{\mathrm{i}}{2} \operatorname{tr}\left(q e_{3} d q^{\dagger}-d q e_{3} q^{\dagger}\right)=d b_{-}^{\dagger} b_{-}-b_{-}^{\dagger} d b_{-}=-d b_{+}^{\dagger} b_{+}+b_{+}^{\dagger} d b_{+}$.

One can easily verify that $\omega=\omega_{j} d x_{j}$ satisfies $\epsilon_{i j k} \partial_{j} \omega_{k}=\partial_{i} \frac{1}{|\vec{x}|}$.

The $U(1)$ is acting by $\mathrm{e}^{\mathrm{i} \phi}:\left(\psi, t_{0 R}\right) \mapsto\left(\psi+2 \phi, t_{0 R}-\phi / l_{R}\right)$. The invariant of this action is $\sigma=\psi+2 l_{R} t_{0 R}$ and the vanishing of the moment maps implies $\vec{t}_{R}=-\frac{1}{2} \vec{x}$. One can readily verify that the above metric becomes

$$
\begin{aligned}
d s^{2}= & \frac{1}{4}\left(\left(l_{R}+\frac{1}{|\vec{x}|}\right) d \vec{x}^{2}+\frac{(d \sigma+\omega)^{2}}{l_{R}+1 /|\vec{x}|}\right)+l_{R} r\left(l_{R}+\frac{1}{|\vec{x}|}\right) \\
& \times\left(d t_{0 R}+\frac{1}{2} \frac{d \sigma+\omega}{l_{R}+1 /|\vec{x}|}\right)^{2} .
\end{aligned}
$$

After factoring out the $\mathrm{e}^{\mathrm{i} \phi}$ action the result is

$$
d s^{2}=\frac{1}{4}\left(\left(l_{R}+\frac{1}{|\vec{x}|}\right) d \vec{x}^{2}+\frac{(d \sigma+\omega)^{2}}{l_{R}+1 /|\vec{x}|}\right) .
$$

The last step in the hyperkähler reduction procedure is the hyperkähler quotient with respect to the $U(1)$ at the distinguished point $s=s_{0}$. In order to represent this action we use the gauge transformation

$$
h(s)= \begin{cases}\exp \left(\mathrm{i} \frac{s}{s_{0}} \varepsilon\right) & \text { for } s \leq s_{0}, \\ \exp \left(\mathrm{i} \frac{l / 2-s}{l / 2-s_{0}} \varepsilon\right) & \text { for } s>s_{0}\end{cases}
$$

that is continuous and equals identity at $s=0$ and at $s=l / 2$. At $s=s_{0}$ this gauge transformation is $h\left(s_{0}\right)=\mathrm{e}^{\mathrm{i} \varepsilon}$. It has the following action:

$$
h(s):\left(\begin{array}{c}
t_{0 L} \\
\vec{t}_{L} \\
t_{0 R} \\
\vec{t}_{R} \\
q
\end{array}\right) \mapsto\left(\begin{array}{c}
t_{0 L}-\varepsilon / s_{0} \\
\vec{t}_{L} \\
t_{0 R}+\varepsilon / l_{R} \\
\vec{t}_{R} \\
q \exp \left(e_{3} \frac{l}{2 s_{0}} \varepsilon\right)
\end{array}\right) .
$$


The corresponding moment map is $\lambda \iota=\frac{l_{L}}{s_{0}} \psi_{L}-\hbar_{R}+\frac{l}{2 s_{0}} \frac{1}{2} \nless$. Since the vanishing of the moment maps of the first stage of the reduction implies $*_{R}=-\frac{1}{2} x$, it follows that $\lambda_{L}=\frac{l_{L}}{s_{0}}\left(t_{L}+\frac{1}{2} x\right)$. Putting $\lambda_{L}$ equal to zero we have $t_{L}=-\frac{1}{2} x$ as well, so $\vec{t}$ is constant on $\mathcal{I}$.

So far, including the data on the left interval, we have the metric

$$
d s^{2}=\frac{1}{4}\left(\left(l_{R}+\frac{1}{|\vec{x}|}\right) d \vec{x}^{2}+\frac{(d \sigma+\omega)^{2}}{l_{R}+1 /|\vec{x}|}\right)+l_{L}\left(d t_{0 L}^{2}+d \vec{t}_{L}^{2}\right) .
$$

Under the above gauge transformation the angle $\sigma=\psi+2 l_{R} t_{0 R} \mapsto \sigma+2 \frac{l_{L}}{s_{0}} \varepsilon$. The invariant coordinate is $\tau=\sigma-2 l_{L} t_{0 L}=\psi+2 l_{R} t_{0 R}-2 l_{L} t_{0 L}$, and we choose $\varepsilon \sim \varepsilon+2 \pi$ instead of $\sigma$ as a coordinate along the circle of the gauge transformation. In these coordinates the above metric can be rewritten as

$$
\begin{aligned}
d s^{2}= & \frac{1}{4}\left[\left(l+\frac{1}{|\vec{x}|}\right) d \vec{x}^{2}+\frac{1}{l+1 /|\vec{x}|}(d \tau+\omega)^{2}\right] \\
& +\frac{l_{L}\left(l+\frac{1}{|\vec{x}|}\right)}{s_{0}^{2}\left(l_{R}+1 /|\vec{x}|\right)}\left(d \varepsilon+\frac{s_{0}}{2} \frac{d \tau+\omega}{(l+1 /|\vec{x}|)}\right)^{2} .
\end{aligned}
$$

The first part of the expression (36) is the resulting hyperkähler metric of the Taub-NUT space

$$
4 d s_{\mathrm{TN}}^{2}=\left(l+\frac{1}{|\vec{x}|}\right) d \vec{r}^{2}+\frac{1}{l+1 /|\vec{x}|}(d \tau+\omega)^{2},
$$

here the one form $\omega$ satisfies $d \omega=*_{3} d \frac{1}{\mid \vec{x}}$. The second part of the expression in equation (36) provides the natural connection $D=d+i s_{0} a$ with the one form $s_{0} a$, where

$$
a=\frac{1}{2} \frac{d \tau+\omega}{l+\frac{1}{|\vec{x}|}}
$$

\subsection{A basis of self-dual two forms}

Let $V=l+1 /|\vec{x}|$, so that $a=\frac{d \tau+\omega}{2 V}$. Here we observe the following relation:

$$
\left(\frac{1}{2} d x-\mathrm{i} a\right)^{\dagger} \wedge\left(\frac{1}{2} d x-\mathrm{i} a\right)=\frac{\mathrm{i}}{2} \sigma_{k}\left(\frac{d \tau+\omega}{V} \wedge d x^{k}+\frac{1}{2} \epsilon_{i j k} d x^{i} d x^{J}\right) .
$$


The components of the right-hand side are self-dual two forms in the orientation $\left(\tau, x^{1}, x^{2}, x^{3}\right)$ providing a basis of self-dual two forms on the Taub-NUT. Let us note for future use that since $\frac{1}{2} d x-\mathrm{i} a=-(d *+\mathrm{i} a)$, in terms of the $\tau$ and $\vec{t}$ coordinates the combination $(d t+\mathrm{i} a)^{\dagger} \wedge(d t+\mathrm{i} a)$ is self-dual.

\section{Instanton data}

Instanton data for an $\mathrm{SU}(2)$ instanton with no monopole charges is represented by the bow diagram in figure 3 . It consists of

- a rank $k_{0}$ vector bundle $E \rightarrow[-l / 2, l / 2]$ with the Nahm data $\left(T_{0}, \vec{T}\right)$ on the intervals $[-l / 2,-\lambda],[-\lambda, \lambda]$, and $[\lambda, l / 2]$ (we do not presume two-sided continuity at $s= \pm \lambda$ across different intervals),

- linear maps $B_{10}: E_{-l / 2} \rightarrow E_{l / 2}$ and $B_{01}: E_{l / 2} \rightarrow E_{-l / 2}$,

- linear maps $I_{L}: W_{L} \rightarrow E_{-\lambda}, J_{L}: E_{-\lambda} \rightarrow W_{L}, I_{R}: W_{R} \rightarrow E_{\lambda}$, and $J_{R}: E_{\lambda} \rightarrow W_{R}$.

The group of gauge transformations acts on these data as follows:

$$
g:\left(\begin{array}{c}
T_{0} \\
T_{j} \\
B_{01} \\
B_{10} \\
I_{\alpha} \\
J_{\alpha}
\end{array}\right) \mapsto\left(\begin{array}{c}
g^{-1}(s) T_{0} g(s)-\mathrm{i}^{-1}(s) \frac{d}{d s} g(s) \\
g^{-1}(s) T_{j} g(s) \\
g^{-1}\left(-\frac{l}{2}\right) B_{01} g\left(\frac{l}{2}\right) \\
g^{-1}\left(\frac{l}{2}\right) B_{10} g\left(-\frac{l}{2}\right) \\
g^{-1}\left(\lambda_{\alpha}\right) I_{\alpha} \\
J_{\alpha} g\left(\lambda_{\alpha}\right)
\end{array}\right),
$$

where the index $\alpha$ takes values $L$ and $R$ and we introduced $\lambda_{L}=-\lambda$ and $\lambda_{R}=\lambda$.

Introducing the complex notation $D=\frac{d}{d s}+\mathrm{i} T_{0}-T_{3}$ and $T=T_{1}+\mathrm{i} T_{2}$, the moment maps are written as

$$
\begin{aligned}
& {[D, T]+\delta\left(s+\frac{l}{2}\right) B_{01} B_{10}-\delta\left(s-\frac{l}{2}\right) B_{10} B_{01}+\sum_{\alpha \in\{L, R\}} \delta\left(s-\lambda_{\alpha}\right) I_{\alpha} J_{\alpha}=0} \\
& {\left[D^{\dagger}, D\right]+\left[T^{\dagger}, T\right]+\delta\left(s+\frac{l}{2}\right)\left(B_{10}^{\dagger} B_{10}-B_{01} B_{01}^{\dagger}\right)+\delta\left(s-\frac{l}{2}\right)\left(B_{01}^{\dagger} B_{01}-B_{10} B_{10}^{\dagger}\right)} \\
& \quad+\sum_{\alpha \in\{L, R\}} \delta\left(s-\lambda_{\alpha}\right)\left(J_{\alpha}^{\dagger} J_{\alpha}-I_{\alpha} I_{\alpha}^{\dagger}\right)=0 .
\end{aligned}
$$




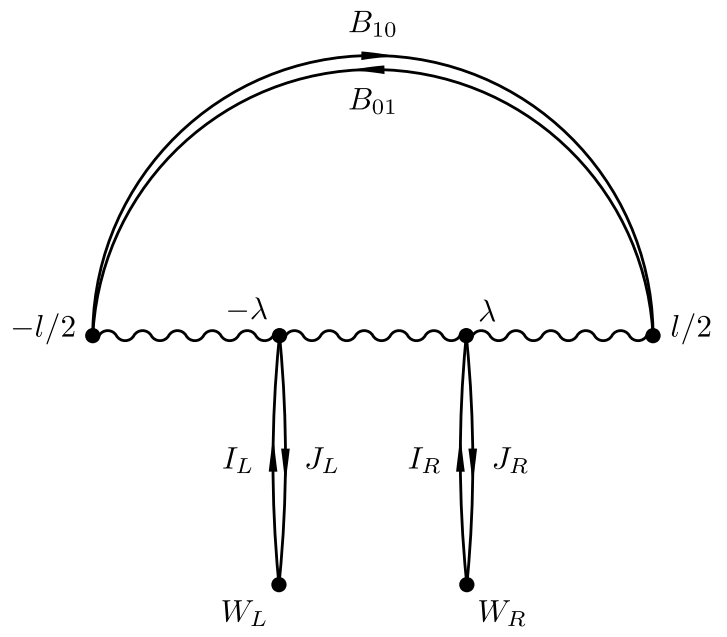

Figure 3: The bow diagram for an SU(2) instanton on the Taub-NUT.

These conditions can be written compactly if we introduce

$$
B_{-}=\left(\begin{array}{c}
B_{10}^{\dagger} \\
-B_{01}
\end{array}\right), \quad B_{+}=\left(\begin{array}{c}
B_{01}^{\dagger} \\
B_{10}
\end{array}\right)
$$

and $\mathcal{D}=-\frac{d}{d s}-\mathrm{i} T_{0}+\mathbb{F}=\left(\begin{array}{cc}-D & T^{\dagger} \\ T & D^{\dagger}\end{array}\right)$. Then the moment maps are given by $\lambda_{l}=\operatorname{Vec}\left(D^{\dagger} D+\sum_{\alpha} \delta\left(s-\lambda_{\alpha}\right) Q_{\alpha} Q_{\alpha}^{\dagger}+\delta\left(s+\frac{l}{2}\right) B_{-} B_{-}^{\dagger}+\delta\left(s-\frac{l}{2}\right) B_{+} B_{+}^{\dagger}\right)$.

\section{The Nahm transform}

\subsection{The Weyl operator}

A central role in the ADHM-Nahm transform $[1,2]$ is played by a certain linear operator. In the case at hand it is a modification of the Weyl operator. The details of similar construction can be found in $[3,25]$ for the case of calorons.

Let $H$ be the space of $L^{2}$ sections of $S \otimes E$ that are continuous on $\mathcal{I}$ and have $L^{2}$ derivatives on $\mathcal{I} \backslash\left\{\lambda_{L}, \lambda_{R}\right\}$. Let $\tilde{\mathcal{H}}$ be the direct sum of the space of $L^{2}$ sections of $S \otimes E$ with spaces $W_{L}, W_{R}, E_{-l / 2}$, and $E_{l / 2}$. Given the 
instanton data of the bow diagram in figure 3 we introduce the operator $\mathfrak{D}: \mathcal{H} \rightarrow \tilde{\mathcal{H}}$ acting by

$$
\mathfrak{D}: f \mapsto\left(\begin{array}{c}
\left(-\frac{d}{d s}-\mathrm{i} T_{0}+\mathbb{T}\right) f \\
\left(J_{L}, I_{L}^{\dagger}\right) f(-\lambda) \\
\left(J_{R}, I_{R}^{\dagger}\right) f(\lambda) \\
\left(B_{01}, B_{10}^{\dagger}\right) f(l / 2) \\
\left(-B_{10}, B_{01}^{\dagger}\right) f(-l / 2)
\end{array}\right) .
$$

Let us denote by $\psi$ an $L^{2}$ section of the restriction of $S \otimes E$ to $\mathcal{I} \backslash\left\{\lambda_{L}, \lambda_{R}\right\}$, $\chi_{\alpha} \in E_{\lambda_{\alpha}}, v_{-} \in E_{-l / 2}$ and $v_{+} \in E_{l / 2}$. Integrating by parts we find that the cokernel of $\mathfrak{D}$ is given by $\left(\psi(s), \chi_{L}, \chi_{R}, v_{-}, v_{+}\right) \in \tilde{\mathcal{H}}$ satisfying

$$
\begin{aligned}
\left(\frac{d}{d s}+\mathrm{i} T_{0}+\mathrm{T}\right) \psi=0, & \text { on } \mathcal{I} \backslash\left\{\alpha_{L}, \alpha_{R}\right\}, \\
\psi\left(\lambda_{\alpha}+\right)-\psi\left(\lambda_{\alpha}-\right) & =-Q_{\alpha} \chi_{\alpha}, \\
\psi(l / 2) & =\left(\begin{array}{c}
B_{01}^{\dagger} \\
B_{10}
\end{array}\right) v_{-} \\
\psi(-l / 2) & =-\left(\begin{array}{c}
-B_{10}^{\dagger} \\
B_{01}
\end{array}\right) v_{+} .
\end{aligned}
$$

In other words, the dual operator takes the form

$$
\begin{aligned}
\mathfrak{D}^{\dagger}= & \left(\begin{array}{cc}
-D^{\dagger} & T^{\dagger} \\
T & D
\end{array}\right) \oplus\left(\underset{\alpha \in\{L, R\}}{\oplus} \delta\left(s-\lambda_{\alpha}\right)\left(\begin{array}{l}
J_{\alpha}^{\dagger} \\
I_{\alpha}
\end{array}\right)\right) \\
& \oplus\left(\delta\left(s+\frac{l}{2}\right)\left(\begin{array}{c}
B_{10}^{\dagger} \\
-B_{01}
\end{array}\right), \delta\left(s-\frac{l}{2}\right)\left(\begin{array}{c}
B_{01}^{\dagger} \\
B_{10}
\end{array}\right)\right), \\
= & D^{\dagger} \oplus \delta\left(s-\lambda_{\alpha}\right) Q_{\alpha} \oplus\left(\delta\left(s+\frac{l}{2}\right) B_{-}, \delta\left(s-\frac{l}{2}\right) B_{+}\right) .
\end{aligned}
$$

In terms of $\mathfrak{D}$ and $\mathfrak{D}^{\dagger}$ the moment map conditions of equation. (41) can be written as

$$
\operatorname{Vec}\left(\mathfrak{D}^{\dagger} \mathfrak{D}\right)=0
$$

For a given point of the Taub-NUT space in figure 2, corresponding to $\left(t_{0}, \vec{t}, b_{10}, b_{01}\right)$ satisfying equation $(23)$, we can twist the above operator as 
follows:

$$
\begin{aligned}
\mathfrak{D}_{t}^{\dagger}= & \left(\begin{array}{cc}
-D^{\dagger}-t_{3} & T^{\dagger}-t^{\dagger} \\
T-t & D+t_{3}
\end{array}\right) \oplus\left(\underset{\alpha \in\{L, R\}}{\oplus} \delta\left(s-\lambda_{\alpha}\right)\left(\begin{array}{c}
J_{\alpha}^{\dagger} \\
I_{\alpha}
\end{array}\right)\right) \\
& \oplus\left(\delta\left(s+\frac{l}{2}\right)\left(\begin{array}{cc}
B_{10}^{\dagger} & -b_{10}^{\dagger} \\
-B_{01} & -b_{01}
\end{array}\right)+\delta\left(s-\frac{l}{2}\right)\left(\begin{array}{cc}
-b_{01}^{\dagger} & B_{01}^{\dagger} \\
b_{10} & B_{10}
\end{array}\right)\right) .
\end{aligned}
$$

To be exact, whenever adding two operators with one of them belonging to the instanton bow and another to the Taub-NUT bow data we understand both operators to be tensored with identity so that they act on the tensor product of the corresponding spaces. For example, $T-t$ stands as a shorthand for $T \otimes 1-1 \otimes t$. Unfortunately, in this case using the rigorous notation would make the formula above much harder to read. We also allow this shorthand since for a case of a single instanton the vector spaces are one dimensional and the bow data are Abelian, so, conveniently, the expression in equation (51) makes perfect sense as it is written.

\subsection{The connection}

From now on we understand $\psi$ to be a section of $\mathbb{C}^{2} \otimes E \otimes e \rightarrow \mathcal{I} \backslash\{-\lambda, \lambda\}$, $v_{-} \in E_{-l / 2} \otimes e_{l / 2}$ and $v_{+} \in E_{l / 2} \otimes e_{-l / 2}$. We combine $v_{+}$and $v_{-}$into a spinor $v=\left(\begin{array}{l}v_{+} \\ v_{-}\end{array}\right)$and denote the data $\left(\psi(s), \chi_{L}, \chi_{R}, v\right)$ by $\boldsymbol{\psi}$. The twisted operator $\mathfrak{D}_{t}^{\dagger}$ acts on the linear Hermitian space formed by such data. For

$$
\boldsymbol{\psi}_{1}=\left(\psi_{1}(s), \chi_{L 1}, \chi_{R 1}^{+}, v_{1}\right) \quad \text { and } \quad \boldsymbol{\psi}_{2}=\left(\psi_{2}(s), \chi_{L 2}, \chi_{R 2}, v_{2}\right)
$$

the natural Hermitian product is given by $\left(\boldsymbol{\psi}_{1}, \boldsymbol{\psi}_{2}\right)=v_{1}^{\dagger} v_{2}+\left(\chi_{L 1}\right)^{\dagger} \chi_{L 2}+$ $\left(\chi_{R 1}\right)^{\dagger} \chi_{R 2}+\int_{-l / 2}^{l / 2} \psi_{1}^{\dagger}(s) \psi_{2}(s) d s$. We also define the operator s acting on $\boldsymbol{\psi}$ as follows:

$$
\mathbf{s}:\left(\psi(s), \chi_{L}, \chi_{R}, v\right) \mapsto\left(s \psi(s),-\lambda \chi_{L}, \lambda \chi_{R},\left(\begin{array}{cc}
-l / 2 & 0 \\
0 & l / 2
\end{array}\right) v\right) .
$$

Once we find the orthonormal basis of solutions of $\mathfrak{D}_{t}^{\dagger} \boldsymbol{\psi}=0$ we arrange them as columns of the matrix $\Psi$, then the orthonormality condition reads $(\Psi, \Psi)=\mathbb{I}$. The instanton connection $\nabla_{\mu}=\partial_{\mu}-\mathrm{i} A_{\mu}$ is induced on the kernel of $\mathfrak{D}_{t}^{\dagger}$ by the connection $D_{\mu}=\partial_{\mu}+$ is $a_{\mu}$, thus, $\nabla_{\mu}=\left(\Psi, D_{\mu} \Psi\right)$ and the 
associated $\mathrm{su}(2)$-valued one form $A=A_{0} d \tau+A_{j} d x^{j}$ is given by

$$
A=\left(\Psi,\left(\mathrm{i} \frac{\partial}{\partial \tau}-\frac{\mathbf{s}}{V}\right) \Psi\right) d \tau+\left(\Psi,\left(\mathrm{i} \frac{\partial}{\partial x_{j}}-\omega_{j} \frac{\mathbf{s}}{V}\right) \Psi\right) d x_{j}
$$

\section{The ADHM limit}

To compare with the ADHM construction we solve $\mathfrak{D}_{t}^{\dagger} \Psi=0$ at the ends of the interval $s= \pm \frac{l}{2}$ to find

$$
\psi\left(-\frac{l}{2}\right)=-\left(\begin{array}{cc}
B_{10}^{\dagger} & -b_{10}^{\dagger} \\
-B_{01} & -b_{01}
\end{array}\right)\left(\begin{array}{c}
c v_{+} \\
v_{-}
\end{array}\right), \quad \psi\left(\frac{l}{2}\right)=\left(\begin{array}{cc}
-b_{01}^{\dagger} & B_{01}^{\dagger} \\
b_{10} & B_{10}
\end{array}\right)\left(\begin{array}{l}
v_{+} \\
v_{-}
\end{array}\right),
$$

The Nahm equations imply that $\vec{t}$ is constant on $[-l / 2, l / 2]$.

It is illustrative to consider first the case of a single instanton. In this case $\vec{T}$ is constant on each of the three intervals of $\mathcal{I} \backslash\{-\lambda, \lambda\}$. Moreover, the values on the left and on the right intervals are equal, thus for some constant vectors $\vec{T}_{1}$ and $\vec{T}_{2}$

$$
\vec{T}(s)= \begin{cases}\vec{T}_{1} & \text { for }-l / 2<s<-\lambda \text { or } \lambda>s>l / 2, \\ \vec{T}_{2} & \text { for }-\lambda<s<\lambda .\end{cases}
$$

Let $\vec{z}_{1}=\vec{t}-\vec{T}_{1}$ and $\vec{z}_{2}=\vec{t}-\vec{T}_{2}$, then the Weyl equation $\mathfrak{D}_{t}^{\dagger} \Psi=0$ becomes equivalent to

$$
\begin{aligned}
& {\left[\mathrm{e}^{-\gtrless_{1}\left(\frac{l}{2}-\lambda_{2}\right)}\left(\begin{array}{cc}
-b_{01}^{\dagger} & B_{01}^{\dagger} \\
b_{10} & B_{10}
\end{array}\right)+\mathrm{e}^{\Varangle_{2}\left(\lambda_{2}-\lambda_{1}\right)} \mathrm{e}^{\Varangle_{1}\left(\lambda_{1}+\frac{l}{2}\right)}\left(\begin{array}{cc}
B_{10}^{\dagger} & -b_{10}^{\dagger} \\
-B_{01} & -b_{01}
\end{array}\right)\right]\left(\begin{array}{c}
v_{+} \\
v_{-}
\end{array}\right)} \\
& +\mathrm{e}^{\vec{\sigma} \cdot \vec{z}_{2}\left(\lambda_{2}-\lambda_{1}\right)}\left(\begin{array}{c}
J_{L}^{\dagger} \\
I_{L}
\end{array}\right) \chi_{L}+\left(\begin{array}{c}
J_{R}^{\dagger} \\
I_{R}
\end{array}\right) \chi_{R}=0 .
\end{aligned}
$$

Clearly in the limit of $l \rightarrow 0$ (and since $\lambda<l / 2$, we have $\lambda \rightarrow 0$ ) the above expression reduces to the ADHM linear equation.

For the case of instantons of general charge the exponentials in the above equation become path-ordered exponentials involving the corresponding nonabelian data $T$. Each of these represents parallel transport along an interval. In the $l \rightarrow 0$ limit, however, all of the intervals in the bow diagram contract to a point and the exponential factors all become identities. Therefore, for a general case, equation (56) for the kernel of $\mathfrak{D}_{t}$ reduces to the ADHM linear equation. 


\section{$7 \quad$ Proof of self-duality}

The core of this proof is close to the original argument of Nahm [2], but requires some adjustments. We shall need the following relations: ${ }^{5}$

$$
(d+\mathrm{i} l a) b_{-}=-\frac{1}{2 t}(d *+\mathrm{i} a) b_{-} \quad \text { and } \quad(d-\mathrm{i} l a) b_{+}=\frac{1}{2 t}(d *+\mathrm{i} a) b_{+} .
$$

We also use the fact that $\mathfrak{D}_{t}^{\dagger} \mathfrak{D}_{t}=1 \otimes \Delta$, with $\Delta$ positive definite (except for some $(\tau, \vec{t})$ corresponding to a finite number of isolated points on the Taub-NUT). Thus, it has a well defined inverse $G=\left(\mathfrak{D}_{t}^{\dagger} \mathfrak{D}_{t}\right)^{-1}$, given by the Green's function of $\Delta$, that commutes with the quaternions and the $\sigma$-matrices.

As expressed by equation (53), the connection induced by $D_{\mu}$ on the kernel of $\mathfrak{D}^{\dagger}$ is the instanton connection $A_{\mu}$, therefore, the covariant differential is $d t^{\mu} \nabla_{\mu} \equiv d t^{\mu}\left(\partial_{\mu}-\mathrm{i} A_{\mu}\right)=P d t^{\mu} D_{\mu} P=P(d+\mathrm{i} s a) P=P\left(d+\mathrm{i} s \frac{d \tau+\omega}{2 V}\right) P$. So the connection is $A_{\mu}=\mathrm{i}\left(\Psi, D_{\mu} \Psi\right)$, then

$$
\begin{aligned}
\partial_{[\mu} A_{\nu]} & =\mathrm{i}\left(D_{[\mu} \Psi, D_{\nu]} \Psi\right)-(\Psi, \mathbf{s} \Psi) \partial_{[\mu} a_{\nu]}, \\
{\left[A_{\mu}, A_{\nu}\right] } & =\left(D_{[\mu} \Psi, \Psi\right)\left(\Psi, D_{\nu]}\right) .
\end{aligned}
$$

It follows that the curvature is

$$
F_{\mu \nu}=\mathrm{i}\left[\nabla_{\mu}, \nabla_{\nu}\right]=\left(D_{[\mu} \Psi,(1-P) D_{\nu]} \Psi\right)-(\Psi, \mathbf{s} \Psi) \partial_{[\mu} a_{\nu]},
$$

where $1-P \equiv 1-\Psi \Psi^{\dagger}=\mathfrak{D}_{t} G \mathfrak{D}_{t}^{\dagger}$. The second term in the curvature expression is self-dual since $d a$ is, while for the first term we have

$$
\left(D_{\mu} \Psi,(1-P) D_{\nu} \Psi\right)=\left(\left[\mathfrak{D}_{t}^{\dagger}, D_{\mu}\right] \Psi, G\left[\mathfrak{D}_{t}^{\dagger}, D_{\nu}\right] \Psi\right)
$$

Since

$$
\begin{aligned}
\mathfrak{D}_{t}^{\dagger}= & \left(\frac{d}{d s}+\mathrm{i} T_{0}+\not{P}-\not\right) \oplus \delta\left(s-\lambda_{\alpha}\right) Q_{\alpha} \\
& \oplus\left(\delta(s+l / 2)\left(B_{-},-b_{+}\right)+\delta(s-l / 2)\left(-b_{-}, B_{+}\right)\right) .
\end{aligned}
$$

\footnotetext{
${ }^{5}$ These follow from equation (30) and $b_{ \pm} b_{ \pm}^{\dagger}=t \pm \hbar$. Namely, (30) implies $4 \mathrm{i} t V a b_{-}=$ $2 b_{-}\left(d b_{-}^{\dagger}\right) b_{-}-b_{-} d\left(b_{-}^{\dagger} b_{-}\right)=2\left(d\left(b_{-} b_{-}^{\dagger}\right)\right) b_{-}-2\left(d b_{-}\right)\left(b_{-}^{\dagger} b_{-}\right)-b_{-} d\left(b_{-}^{\dagger} b_{-}\right)=2(d t-d \hbar) b_{-}-$ $4 t d b_{-}-2 d t b_{-}=-4 t d b_{-}-2 d k b_{-}$, thus, $2 t\left(d b_{-}+\mathrm{i} l a b_{-}\right)=-(d *+\mathrm{i} a) b_{-}$.
} 
The commutator $d t^{\nu}\left[\mathfrak{D}_{t}^{\dagger}, D_{\nu}\right]$ is given by

$$
\begin{aligned}
{\left[\mathfrak{D}_{t}^{\dagger}, d+\mathrm{i} \mathbf{s} a\right] } & =(d t+\mathrm{i} a) \oplus 0 \oplus\left(\delta(s-l / 2)(d+\mathrm{i} l a) b_{-}, \delta(s+l / 2)(d-\mathrm{i} l a) b_{+}\right) \\
& =(d t+\mathrm{i} a)\left(1 \oplus 0 \oplus\left(-\frac{1}{2 t} \delta(s-l / 2) b_{-}, \frac{1}{2 t} \delta(s+l / 2) b_{+}\right)\right) .
\end{aligned}
$$

As the Green's function $G=\left(\mathfrak{D}^{\dagger} \mathfrak{D}\right)^{-1}$ is scalar, that is, it commutes with the $\sigma$-matrices, and $(d *-\mathrm{i} a) \wedge(d *+\mathrm{i} a)=\left(\frac{1}{2} d x+\mathrm{i} a\right) \wedge\left(\frac{1}{2} d x-\mathrm{i} a\right)$ is selfdual according to equation (39), the curvature two form $F=F_{\mu \nu} d x^{\mu} d x^{\nu}$ is self-dual as well due to equations (60),(61) and (63).

\section{Instantons for the $U(n)$ gauge group}

Generalizing our construction to instantons with the gauge group $U(n)$ is fairly straightforward. The corresponding bow diagram is given in figure 4 . The positions of the marked points $\lambda_{1}, \ldots, \lambda_{n}$ partitioning the interval $[-l / 2$, $l / 2$ ] are given by the asymptotic of the eigenvalues of the instanton connection monodromy around the Taub-NUT circle. All of our previous discussion including the proof of the self-duality and the ADHM limit remains valid.

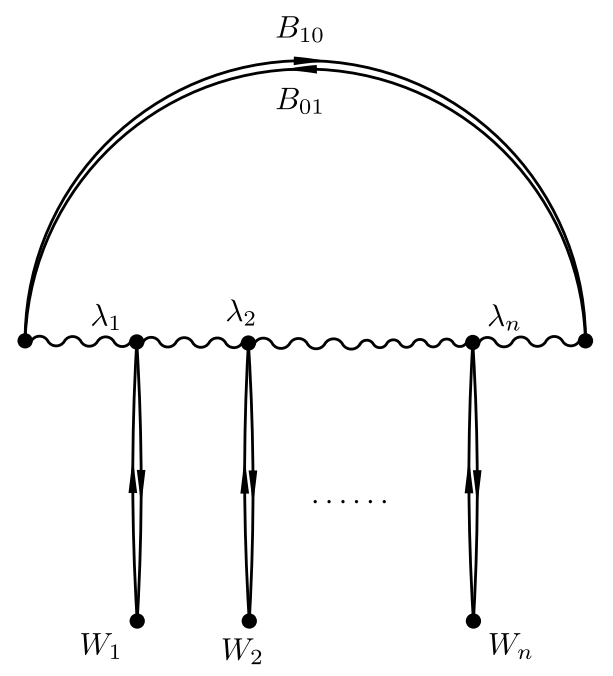

Figure 4: Bow diagram for $U(n)$ instanton on Taub-NUT. 


\section{The geometric meaning of the Nahm transform for curved manifolds}

The conventional Nahm transform [26] of some self-dual configuration (or of a dimensional reduction of a self-dual configuration) on a flat manifold $M=\mathbb{R}^{4} / \Lambda$ results in some data on a dual space $N$ of flat connections on $M$. The kernel of the Nahm transform is the Poincaré bundle $\mathfrak{P} \rightarrow M \times N$. Let us denote the two projections of the product $M \times N$ on $M$ and $N$ by $p_{M}$ and $p_{N}$ respectively, so that we have the following diagram:

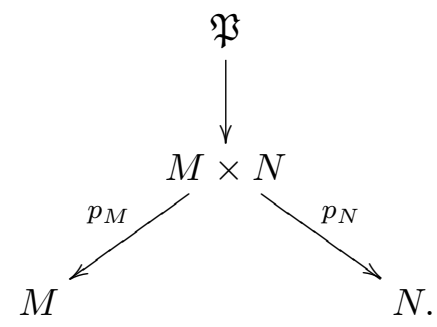

Then for an instanton bundle $\mathcal{E} \rightarrow M$ its Nahm transform is $p_{N *}\left(\mathfrak{P} \otimes p_{M}^{*} \mathcal{E}\right)$. Thus, the Poincaré bundle $\mathfrak{P}$ plays the role of the kernel of this transform.

For example, for the case of a caloron, flat connections on $\mathbb{R}^{3} \times S^{1}$ have the form $\eta=s d t_{0}$, where $t_{0}$ is the coordinate parameterizing the $S^{1}$ factor. The space of such connections forms the dual circle $\hat{S}^{1}$ parameterized by $s$. The Poincaré bundle over the product $\left(\mathbb{R}^{3} \times S^{1}\right) \times \hat{S}^{1}$ has a natural connection $\eta$ with curvature $\mathcal{F}=d s \wedge d t_{0}$, and it can be trivialized on either one of the two base components, making both pushforward operations $p_{N *}$ and $p_{M *}$ simple and well defined.

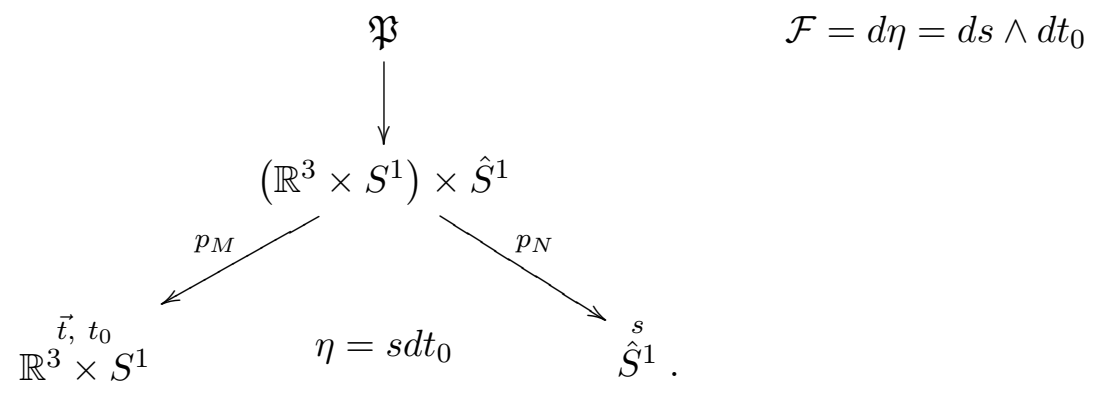

For a curved manifold $M$ without flat connections, such as the Taub-NUT space, a generalization of the Nahm transform is less straightforward. In 
order to have a version of the Nahm transform in the diagram (66) below, one has to answer two questions: (1) What is the correct choice of the "dual" manifold $N$ ? and (2) What is the kernel $\mathfrak{M}$ generalizing the Poincaré bundle?

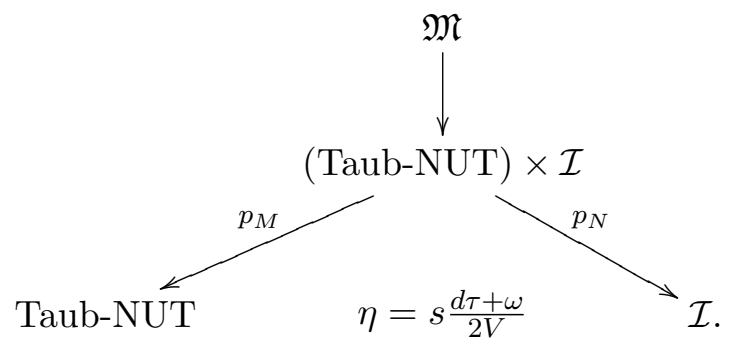

We propose that for the Taub-NUT space the appropriate choice of $N$ is the space $\mathcal{I}$ of self-dual Abelian connections on the Taub-NUT (or rather, in order to have a hyperkähler space, the direct product of $\mathcal{I}$ and $\mathbb{R}^{3}$ ). In order to answer the second question, we digress to discuss a generalization of instantons in four dimensions to instantons on higher-dimensional spaces.

Instantons on higher-dimensional hyperkähler manifolds were defined in [27] in the following manner. Consider the operator $\aleph=I \otimes I+J \otimes$ $J+K \otimes K$ acting on two forms. Due to the defining quaternionic identities it satisfies

$$
\aleph^{2}=2 \aleph+3,
$$

and can have eigenvalues 3 or -1 . On a four-dimensional hyperkähler manifold this operator is related to the Hodge star operation by $*=\frac{1}{2}(\aleph-1)$, thus on a general hyperkähler manifold the equations

$$
\aleph \mathcal{F}=3 \mathcal{F} \quad \text { and } \quad \aleph \mathcal{F}=-\mathcal{F},
$$

respectively, generalize the self-duality and anti-self-duality conditions to higher dimensions.

Before we proceed, let us observe, that the complex structures $I, J$, and $K$ act on the vierbein $\mathrm{e}^{\hat{\mu}}$ of the Taub-NUT

$$
\mathrm{e}^{\hat{0}}=\frac{1}{2 \sqrt{V}}(d \tau+\omega), \quad \mathrm{e}^{\hat{j}}=\frac{1}{2} \sqrt{V} d x^{\hat{j}},
$$

by acting with the left multiplication on the quaternionic combination $\mathrm{e}^{\hat{0}}+I \mathrm{e}^{\hat{1}}+J \mathrm{e}^{\hat{2}}+K \mathrm{e}^{\hat{3}}$. 
Just as the four-dimensional self-duality equations become Bogomolny equations under the reduction to three dimensions, we reduce an eightdimensional self-duality condition $3 \mathcal{F}=\aleph \mathcal{F}$ to five dimensions producing the following system of equations on $\mathcal{I} \times$ Taub-NUT:

$$
\begin{gathered}
3 \mathcal{F}_{\hat{0} s}=\hat{D}_{1} \Phi_{1}+\hat{D}_{2} \Phi_{2}+\hat{D}_{3} \Phi_{3}, \\
3 \mathcal{F}_{\hat{1} s}=-\hat{D}_{0} \Phi_{1}-\hat{D}_{3} \Phi_{2}+\hat{D}_{2} \Phi_{3}, \\
3 \mathcal{F}_{\hat{2} s}=\hat{D}_{3} \Phi_{1}-\hat{D}_{0} \Phi_{2}-\hat{D}_{1} \Phi_{3}, \\
3 \mathcal{F}_{\hat{3} s}=-\hat{D}_{2} \Phi_{1}+\hat{D}_{1} \Phi_{2}-\hat{D}_{0} \Phi_{3}, \\
2 \mathcal{F}_{\hat{\mu} \hat{\nu}}=\varepsilon_{\hat{\mu} \hat{\nu} \hat{\rho} \hat{\sigma}} \mathcal{F}_{\hat{\rho} \hat{\sigma}} .
\end{gathered}
$$

Here $\Phi_{1}, \Phi_{2}, \Phi_{3}$ are the components of the eight-dimensional connection in the reduced three directions of $\mathbb{R}^{3}$. We used the curvature vierbein components $\mathcal{F}=\mathcal{F}_{s \hat{\rho}} d s \wedge \mathrm{e}^{\hat{\rho}}+\mathcal{F}_{\hat{\mu} \hat{\nu}} \mathrm{e}^{\hat{\mu}} \wedge \mathrm{e}^{\hat{\nu}}$ and $\hat{D}_{0}=2 \sqrt{V} D_{0}$ and $\hat{D}_{j}=\frac{2}{\sqrt{V}}$ $D_{j}-4 \omega_{j} D_{0}$, which appear in the covariant differential decomposition $D=d \tau D_{0}+d x^{j} D_{j}=\mathrm{e}_{\hat{0}} \hat{D}_{\hat{0}}+\mathrm{e}^{\hat{j}} \hat{D}_{\hat{j}}$.

Since equations (9) emerge via a dimensional reduction of higherdimensional self-duality equations, one might call an object satisfying these equations an Instapole or a Monotone. ${ }^{6}$

In our case $\mathcal{F}=d \eta=d\left(s \frac{d \tau+\omega}{2 V}\right)=d s \wedge a+s d a=\frac{1}{\sqrt{V}} d s \wedge \mathrm{e}^{0}+s d a$ as dictated by relation (65) between $\mathcal{I}$ and the Taub-NUT. We observe that $\Phi_{1}=t_{1}=-\frac{1}{2} x_{1}, \Phi_{2}=t_{2}=-\frac{1}{2} x_{2}, \Phi_{3}=t_{3}=-\frac{1}{2} x_{3}$ augment $\mathcal{F}$ to produce a solution to the system of equation (9). This is exactly the solution defining the object generalizing the Poincaré bundle in diagram (66) that leads to the twisting that we used in equation (51). It plays the role of the kernel in this generalization of the Nahm transform.

\section{Example of one instanton}

Let us now focus on a single SU(2) instanton on the Taub-NUT, i.e., a selfdual curvature configuration with $k_{0}=1$ and $m=0$. For a single instanton the T's in the Nahm data are Abelian and the Nahm equations are solved by

$$
\vec{T}(s)= \begin{cases}\vec{T}_{1} & \text { for }-l / 2<s<-\lambda \quad \text { or } \quad \lambda>s>l / 2 \\ \vec{T}_{2} & \text { for }-\lambda<s<\lambda\end{cases}
$$

\footnotetext{
${ }^{6}$ We hope someone will come up with a more poetic name for it.
} 
We interpret $\vec{x}=-2 \vec{T}_{1}$ and $\vec{x}=-2 \vec{T}_{2}$ as the locations of the instanton constituents. Let $\vec{z}_{1}=\vec{t}-\vec{T}_{1}$ and $\vec{z}_{2}=\vec{t}-\vec{T}_{2}$ denote the position relative to the two constituents and let $\vec{y}=\vec{T}_{2}-\vec{T}_{1}=\vec{z}_{1}-\vec{z}_{2}$ be the displacement between them. The $\tau$ coordinate of the instanton position is proportional to $T_{0}$. Since the Taub-NUT metric is invariant with respect to shifts of $\tau$, without loss of generality $T_{0}$ can be put to zero. We also gauge away $t_{0}$ in favor of the phase of $b_{ \pm}$. Let the two-component spinors $Q_{+}$and $Q_{-}$be such

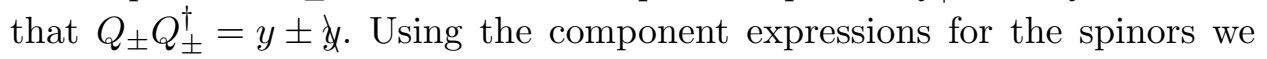
introduced earlier

$$
b_{-}=\left(\begin{array}{c}
b_{01}^{\dagger} \\
-b_{10}
\end{array}\right), \quad b_{+}=\left(\begin{array}{c}
b_{10}^{\dagger} \\
b_{01}
\end{array}\right), \quad B_{-}=\left(\begin{array}{c}
B_{10}^{\dagger} \\
-B_{01}
\end{array}\right), \quad B_{+}=\left(\begin{array}{c}
B_{01}^{\dagger} \\
B_{10}
\end{array}\right),
$$

and, since in this case all of the components are simply complex numbers, it is straightforward to verify that

$$
b_{-}^{\dagger} B_{-}=B_{+}^{\dagger} b_{+}=\mathrm{e}^{\mathrm{i} \tau / 2} \mathcal{P}, \quad b_{+}^{\dagger} B_{+}=B_{-}^{\dagger} b_{-}=\mathrm{e}^{-\mathrm{i} \tau / 2} \mathcal{P},
$$

where

$$
\mathcal{P}=\sqrt{\left(T_{1}+t\right)^{2}-z_{1}^{2}}
$$

The moment maps at $s= \pm l / 2$ imply that

$$
b_{ \pm} b_{ \pm}^{\dagger}=|\vec{t}| \pm \hbar, \quad B_{ \pm} B_{ \pm}^{\dagger}=\left|\vec{T}_{1}\right| \pm \bigvee_{1},
$$

and the vanishing of the moment maps at $s= \pm \lambda$ implies $Q_{R}=Q_{+}$and $Q_{L}=Q_{-}$.

\subsection{Solving the Weyl equation}

On each interval $\mathfrak{D}_{t}=-\partial_{s}+\mathbb{T}-\sharp=-\partial_{s}-\Varangle$ and $\mathfrak{D}_{t}^{\dagger}=\partial_{s}+\mathbb{T}-\not=\partial_{s}-$ $£$, with $\Varangle=\Varangle_{1}$ or $\Varangle_{2}$ in accordance with equation (55). It follows therefore, that $\psi(s)$ has the form

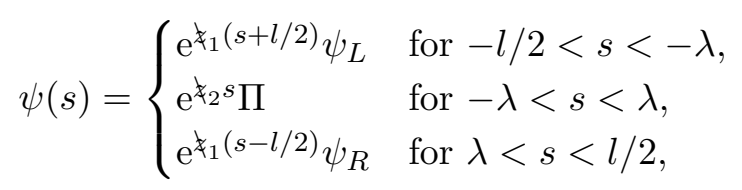

for some constant $\psi_{L}, \psi_{R}$, and $\Pi$. As we shall soon verify, the kernel of $\mathfrak{D}_{t}^{\dagger}$ is two dimensional, so from now on we shall understand $\psi(s), \chi_{L}, \chi_{R}$ and 
$v$ to be two-column matrices, so that their first columns deliver one of the solutions and their second columns deliver the second, linearly independent, solution of $\mathfrak{D}_{t}^{\dagger} \boldsymbol{\psi}=0$. read

Let $A_{L}=\left(B_{-},-b_{+}\right)$and $A_{R}=\left(-b_{-}, B_{+}\right)$then the $\mathfrak{D}_{t}^{\dagger} \Psi=0$ conditions

$$
\begin{gathered}
\psi_{R}-A_{R} v=0 \quad \text { at } s=\frac{l}{2}, \\
\mathrm{e}^{-\Varangle_{1}(l / 2-\lambda)} \psi_{R}-\mathrm{e}^{\Varangle_{2} \lambda} \Pi+Q_{R} \chi_{R}=0 \quad \text { at } s=\lambda, \\
\mathrm{e}^{-\Varangle_{2} \lambda} \Pi-\mathrm{e}^{\Varangle_{1}(l / 2-\lambda)} \psi_{L}+Q_{L} \chi_{L}=0 \quad \text { at } s=-\lambda, \\
\psi_{L}+A_{L} v=0 \quad \text { at } s=-\frac{l}{2} .
\end{gathered}
$$

It is useful to note the following relations:

$$
\begin{aligned}
A_{L} A_{L}^{\dagger} & =T_{1}+t+\Varangle_{1}, \quad A_{R}^{\dagger} A_{L}=A_{L} A_{R}^{\dagger}=-\mathrm{e}^{\mathrm{i} \tau / 2} \mathcal{P}, \\
A_{L} A_{R}^{-1} & =-\frac{\mathrm{e}^{\mathrm{i} \tau / 2}}{\mathcal{P}}\left(T_{1}+t+\Varangle_{1}\right), \quad A_{R} A_{R}^{\dagger}=T_{1}+t-\Varangle_{1}, \\
A_{L}^{\dagger} A_{R} & =A_{R} A_{L}^{\dagger}=-\mathrm{e}^{-\mathrm{i} \tau / 2} \mathcal{P}, \quad A_{R} A_{L}^{-1}=-\frac{\mathrm{e}^{-\mathrm{i} \tau / 2}}{\mathcal{P}}\left(T_{1}+t-\Varangle_{1}\right),
\end{aligned}
$$

and define $\mu_{+}$and $\mu_{-}$to be such that $\mu_{+}^{2}=A_{L} A_{L}^{\dagger}$ and $\mu_{-}^{2}=A_{R} A_{R}^{\dagger}$ namely

$$
\mu_{ \pm}=\sqrt{\frac{T_{1}+t+\mathcal{P}}{2}} \pm \sqrt{\frac{T_{1}+t-\mathcal{P}}{2}} \frac{\Varangle_{1}}{z_{1}},
$$

then $\mu_{+} \mu_{-}=\mathcal{P}$.

We choose

$$
v=-\mathrm{e}^{\mathrm{i} \tau / 4} A_{L}^{\dagger} \frac{\mu_{-}}{\mathcal{P}}=\mathrm{e}^{-\mathrm{i} \tau / 4} A_{R}^{\dagger} \frac{\mu_{+}}{\mathcal{P}},
$$

so that now $\psi_{L}=\mathrm{e}^{\mathrm{i} \tau / 4} \mu_{+}, \psi_{R}=\mathrm{e}^{-\mathrm{i} \tau / 4} \mu_{-}$. From the matching conditions equations (78) and (79) at $s= \pm \lambda$ it follows that

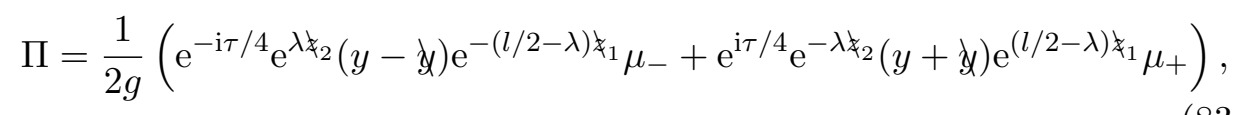


where the function $g$ is given by

$$
g=y \cosh 2 z_{2} \lambda-\frac{\vec{z}_{2} \cdot \vec{y}}{z_{2}} \sinh 2 z_{2} \lambda=\frac{1}{2}\left(\mathrm{e}^{2 \Varangle_{2} \lambda} Q_{-} Q_{-}^{\dagger}+Q_{+} Q_{+}^{\dagger} \mathrm{e}^{-2 \Varangle_{2} \lambda}\right),
$$

and that

$$
\left(\begin{array}{c}
\chi_{R} \\
\chi_{L}
\end{array}\right)=\left(\begin{array}{c}
Q_{+}^{\dagger} \mathrm{e}^{-\lambda \Varangle_{2}} \\
Q_{-}^{\dagger} \mathrm{e}^{\lambda \Varangle_{2}}
\end{array}\right) \Upsilon
$$

with

$$
\Upsilon=\frac{\mathrm{e}^{\mathrm{i} \tau / 4} \mathrm{e}^{\lambda \Varangle_{2}} \mathrm{e}^{\left(\frac{l}{2}-\lambda\right) \Varangle_{1}} \mu_{+}-\mathrm{e}^{-\mathrm{i} \tau / 4} \mathrm{e}^{-\lambda \Varangle_{2}} \mathrm{e}^{-\left(\frac{l}{2}-\lambda\right) \Varangle_{1}} \mu_{-}}{2 g} .
$$

\subsection{Normalization}

Let us now check the orthogonality and the normalization of the solution $\Psi$ delivered by equations (76), (82) and (85). To simplify our notation let us introduce $\alpha=\frac{1}{4 z_{1}} \ln \frac{T_{1}+t+z_{1}}{T_{1}+t-z_{1}}$, so that $\mu_{-}^{2}=T_{1}+t-\Varangle_{1}=\mathcal{P e}^{-2 \alpha \S_{1}}$, and in particular that $\sinh 2 \alpha z_{1}=z_{1} / \mathcal{P}$ and $\cosh 2 \alpha z_{1}=\left(T_{1}+t\right) / \mathcal{P}$. Introduce $\Delta=\frac{l}{2}-\lambda+\alpha$ and let

$$
\begin{gathered}
c_{1}=\cosh 2 \Delta z_{1}=\frac{\left(T_{1}+t\right) \cosh (l-2 \lambda) z_{1}+z_{1} \sinh (l-2 \lambda) z_{1}}{\mathcal{P}} \\
s_{1}=\sinh 2 \Delta z_{1}=\frac{z_{1} \cosh (l-2 \lambda) z_{1}+\left(T_{1}+t\right) \sinh (l-2 \lambda) z_{1}}{\mathcal{P}} \\
c_{2}=\cosh 2 \lambda z_{2}, \quad s_{2}=\sinh 2 \lambda z_{2}
\end{gathered}
$$

In these terms $g=y c_{2}+\frac{y^{2}+z_{2}^{2}-z_{1}^{2}}{2 z_{2}} s_{2}$. Then we find that $(\Psi, \Psi)=N^{2} \mathbb{I}_{2 \times 2}$ with the normalization factor

$$
N^{2}=(\Psi, \Psi)=\frac{\mathcal{P}}{g}\left(c_{1} c_{2}+\frac{y}{z_{1}} s_{1} c_{2}+\frac{y}{z_{2}} c_{1} s_{2}+\frac{z_{1}^{2}+z_{2}^{2}+y^{2}}{2 z_{1} z_{2}} s_{1} s_{2}-\cos \frac{\tau}{2}\right)
$$

Let us also observe that both $\Pi$ and $\Upsilon$ appearing in the solution are scalar multiples of unitary matrices, since

$$
\Pi^{\dagger} \Pi=\frac{\mathcal{P}}{g}\left(y c_{1}+\frac{\vec{y} \cdot \vec{z}_{1}}{z_{1}} s_{1}\right), \quad \Upsilon^{\dagger} \Upsilon=\frac{\mathcal{P}}{2 g^{2}}\left(c_{1} c_{2}+s_{1} s_{2} \frac{\overrightarrow{z_{2}} \cdot \overrightarrow{z_{1}}}{z_{1} z_{2}}-\cos \frac{\tau}{2}\right)
$$




\subsection{Connection}

We rewrite equation (53) as $A=A^{(0)} d \tau+A^{(3)}-\Phi \frac{1}{2 V}(d \tau+\vec{\omega} \cdot d \vec{x})$, where

$$
\Phi=\left(\Psi_{N}, \mathbf{s} \Psi_{N}\right), \quad A^{(0)}=\mathrm{i}\left(\Psi_{N}, \frac{\partial}{\partial \tau} \Psi_{N}\right), \quad A^{(3)}=\mathrm{i}\left(\Psi_{N}, \frac{\partial}{\partial x_{j}} \Psi_{N}\right) d x^{j},
$$

for an orthonormalized solution $\Psi_{N}$ satisfying $\left(\Psi_{N}, \Psi_{N}\right)=\mathbb{I}_{2 \times 2}$. Here we observe that for any $\Psi=N \Psi_{N}$, with $N$ any nowhere vanishing scalar function, we have

$$
\begin{aligned}
\left(\Psi_{N}, \frac{\partial}{\partial x^{\mu}} \Psi_{N}\right) & =\frac{1}{2}\left(\left(\Psi_{N}, \frac{\partial}{\partial x^{\mu}} \Psi_{N}\right)-\left(\frac{\partial}{\partial x^{\mu}} \Psi_{N}, \Psi_{N}\right)\right) \\
& =\frac{1}{2 N^{2}}\left(\left(\Psi, \frac{\partial}{\partial x^{\mu}} \Psi\right)-\left(\frac{\partial}{\partial x^{\mu}} \Psi, \Psi\right)\right) .
\end{aligned}
$$

Thus for the solution of Section 10.1 which satisfies $(\Psi, \Psi)=N^{2} \mathbb{I}_{2 \times 2}$ we have

$$
\begin{gathered}
\Phi=\frac{1}{N^{2}}(\Psi, \mathbf{s} \Psi) \\
A^{(0)}=\frac{\mathrm{i}}{N^{2}}\left(\Psi, \frac{\partial}{\partial \tau} \Psi\right)=\frac{\mathrm{i}}{2 N^{2}}\left(\left(\Psi, \frac{\partial}{\partial \tau} \Psi\right)-\left(\frac{\partial}{\partial \tau} \Psi, \Psi\right)\right) \\
A^{(3)}=\frac{\mathrm{i}}{N^{2}}(\Psi, d \Psi)=\frac{\mathrm{i}}{2 N^{2}}(\Psi,(d-\overleftarrow{d}) \Psi) \equiv \frac{\mathrm{i}}{2 N^{2}}((\Psi, d \Psi)-(d \Psi, \Psi))
\end{gathered}
$$

where we introduced the three-dimensional differential $d=d x^{j} \frac{\partial}{\partial x^{j}}=d t^{j} \frac{\partial}{\partial t^{j}}$.

Given our solution for $\Psi$ of equations (76), (82) and (85) one can apply the above formulas, performing some elementary integrals over $s$. A straightforward if tedious calculation gives

$$
\begin{aligned}
& N^{2} A^{(0)}=\frac{1}{4}\left(2 t-c_{1} \mathcal{P}\right) \frac{\varliminf_{1}}{z_{1}^{2}}+\frac{1}{2 \mathcal{P}}\left(F_{1}-\frac{\vec{T}_{1} \cdot \vec{z}_{1}}{z_{1}} \frac{\varliminf_{1}}{z_{1}}\right) \\
& +\frac{i}{2} \frac{s_{2}}{z_{2}} \Pi^{\dagger}\left(\partial_{\tau}-\overleftarrow{\partial}_{\tau}\right) \Pi+i g \Upsilon^{\dagger}\left(\partial_{\tau}-\overleftarrow{\partial}_{\tau}\right) \Upsilon \\
& N^{2} \Phi=\left(1+2 l t-\left(2 \lambda c_{1}+\frac{s_{1}}{z_{1}}\right) \mathcal{P}\right) \frac{\varliminf_{1}}{2 z_{1}^{2}}+\frac{l}{\mathcal{P}}\left(F_{1}-\frac{F_{1} \cdot \varliminf_{1}}{z_{1}} \frac{\varliminf_{1}}{z_{1}}\right)
\end{aligned}
$$

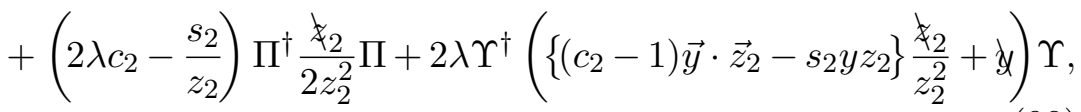




$$
\begin{aligned}
& N^{2} A^{(3)}=\frac{\mathrm{i}}{2}\left\{\frac{z_{1}}{\mathcal{P}^{2}}\left[F_{1}, d \frac{\aleph_{1}}{z_{1}}\right]+\frac{1}{\mathcal{P}^{3}}\left(\frac{T_{1}+t}{z_{1}} \frac{\vec{z}_{1} \cdot d \vec{t}}{z_{1}}-\frac{\vec{t} \cdot d \vec{t}}{t}\right)\left[F_{1}, \gtrless_{1}\right]\right. \\
& -\left(1+\mathcal{P}\left(l-2 \lambda-\frac{s_{1}}{z_{1}}\right)-2 \frac{T_{1}\left(T_{1}+t-\mathcal{P}\right)}{\mathcal{P}^{2}}\right) \frac{\left[\S_{1}, d \hbar\right]}{2 z_{1}^{2}} \\
& +\Pi^{\dagger}\left(\frac{s_{2}}{z_{2}} d-\overleftarrow{d} \frac{s_{2}}{z_{2}}-\left(2 \lambda-\frac{s_{2}}{z_{2}}\right) \frac{\left[£_{2}, d \star\right]}{2 z_{2}^{2}}\right) \Pi
\end{aligned}
$$

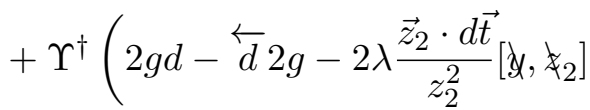

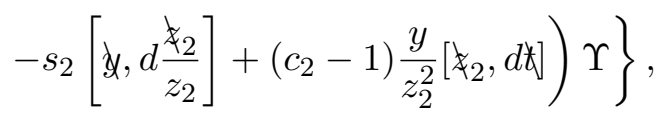

where the functions $\mathcal{P}$ and $g$ are defined in equations (74) and (84), the hyperbolic functions $c_{1}, s_{1}, c_{2}$ and $s_{2}$ are in equations (74), (88) and (89), and $\Pi$ and $\Upsilon$ are given in equations (83) and (86). The normalization factor $N^{2}$ is read from equations $(90)$.

\section{Conclusions}

We discussed topological charges of an instanton configuration on the TaubNUT space with the maximal symmetry breaking by the monodromy at infinity. These are given by integer monopole charges and an integer instanton number. Solutions with vanishing instanton number correspond to singular monopoles [18]. In their three-dimensional interpretation these have infinite energy, while as configurations on the Taub-NUT space they are smooth and have finite action. Thus one can regard the Taub-NUT background as a regularization. A simplest solution with zero instanton number was constructed in [21] and its physical properties were explored in [22].

In this manuscript we focussed on the case with vanishing monopole charges. We presented the ADHM-Nahm data for this case. These data are conveniently encoded in a bow diagram, such as in figures 3 or 4 . We used the bow diagram description earlier in [16] to study the moduli spaces of instantons on the Taub-NUT. Here we give the details of the Nahm transform leading to the explicit instanton connection.

As an example illustrating this construction we find a single SU(2) instanton on the Taub-NUT space in equations (97)-(99).

The bow diagram formalism we presented is not limited to the case of the Taub-NUT background. Rather, we chose to limit the scope of this paper to this case to simplify our presentation. In the forthcoming paper [28] we will 
give the bow-diagrammatic description of instantons with arbitrary charges on a general ALF spaces of either $A_{k^{-}}$or $D_{k^{-}}$type.

\section{Acknowledgments}

It is our pleasure to thank Tamas Hausel and Juan Maldacena for a number of useful conversations. We are grateful to Christopher Blair for idenitfying a number of misprints in the original version of this paper. This work is supported by Science Foundation Ireland Grant No. 06/RFP/MAT050 and by the European Commission FP6 program MRTN-CT-2004-005104.

This work is dedicated to the memory of Raphael Siev.

\section{Appendix A.}

\section{A.1 Metric conventions and moment maps}

The Nahm data on an interval of length $l$ can be organized into a quaternion $t=t_{0} e_{0}+\vec{t} \cdot \vec{e}$ with the metric and the symplectic forms

$$
\begin{aligned}
d s^{2} & =g(\cdot, \cdot)=l \frac{1}{2} \operatorname{tr} d t d t^{\dagger}, \omega_{j}(A, B):=g\left(A, e_{j} B\right)=-\frac{l}{4} \operatorname{tr} e_{j}\left(A B^{\dagger}-B A^{\dagger}\right) \\
\omega_{j} & =g\left(\cdot, e_{j} \cdot\right)=-\frac{l}{4} \operatorname{tr}\left(d t \wedge d t^{\dagger} e_{j}\right)
\end{aligned}
$$

With respect to $t \mapsto t+\epsilon$ the moment maps are $\mu_{j}=-\frac{l}{4} \operatorname{tr}\left(t^{\dagger}-t\right) e_{j}=l t_{j}$.

For $q=\left(b_{-}, b_{+}\right)$the metric is $d s^{2}=\frac{1}{2} \operatorname{tr} d q d q^{\dagger}=d b_{-}^{\dagger} d b_{-}=d b_{+}^{\dagger} d b_{+}$. The moment map with respect to $q \mapsto q \mathrm{e}^{\epsilon e_{3}}$ is $\mu_{j}=-\frac{1}{4} \operatorname{tr} q \mathrm{e}_{3} q^{\dagger} \mathrm{e}_{j}$.

Coordinates on Taub-NUT are either $b_{+}, b_{-}$or $\vec{t}, 2 l t_{0}=\tau \sim \tau+4 \pi$. The instanton moduli are $\vec{T}_{1}, \theta_{1}=(2 l-4 \lambda) T_{0}^{L}$ (or $B_{+}, B_{-}$) and $\vec{T}_{2}, \theta_{2}=4 \lambda T_{0}^{M}$. The relative coordinates are $\vec{z}_{1}=\vec{t}-\vec{T}_{1}, \vec{z}_{2}=\vec{t}-\vec{T}_{2}$, and the relative position is $\vec{y}=\vec{T}_{2}-\vec{T}_{1}=\vec{z}_{1}-\vec{z}_{2}$.

We also collect the bifundamental data as

$$
b_{-}=\left(\begin{array}{c}
b_{01}^{\dagger} \\
-b_{10}
\end{array}\right), \quad b_{+}=\left(\begin{array}{c}
b_{10}^{\dagger} \\
b_{01}
\end{array}\right), \quad B_{-}=\left(\begin{array}{c}
B_{10}^{\dagger} \\
-B_{01}
\end{array}\right), \quad B_{+}=\left(\begin{array}{c}
B_{01}^{\dagger} \\
B_{10}
\end{array}\right)
$$


and the fundamental data as

$$
Q_{-}=\left(\begin{array}{c}
J_{L}^{\dagger} \\
I_{L}
\end{array}\right), Q_{+}=\left(\begin{array}{c}
J_{R}^{\dagger} \\
I_{R}
\end{array}\right)
$$

\section{A.2 Vanishing moment map conditions}

For the Taub-NUT

$$
\frac{d}{d s} \hbar+\operatorname{Vec}\left\{\delta\left(s+\frac{l}{2}\right) b_{-} b_{-}^{\dagger}+\delta\left(s-\frac{l}{2}\right) b_{+} b_{+}^{\dagger}\right\}=0
$$

and for the instanton Bow data

$$
\begin{aligned}
& {\left[\frac{d}{d s}+\mathrm{i} T_{0}, F\right]+\operatorname{Vec}\left\{F \bar{F}+\delta\left(s+\frac{l}{2}\right) B_{-} B_{-}^{\dagger}+\delta\left(s-\frac{l}{2}\right) B_{+} B_{+}^{\dagger}\right.} \\
& \left.\quad+\delta(s+\lambda) Q_{-} Q_{-}^{\dagger}+\delta(s-\lambda) Q_{+} Q_{+}^{\dagger}\right\}=0
\end{aligned}
$$

These imply that $\vec{T}$ is constant on each interval and equals $\vec{T}_{1}$ for $|s|>\lambda$ and $\vec{T}_{2}$ for $|s|<\lambda$. The conditions at $s=l / 2,-l / 2, \lambda,-\lambda$ are respectively

$T_{1}+T_{1}=B_{+} B_{+}^{\dagger}, \quad T_{1}-T_{1}=B_{-} B_{-}^{\dagger}, \quad y+\phi_{1}=Q_{+} Q_{+}^{\dagger}, \quad y-\dot{y}=Q_{-} Q_{-}^{\dagger}$.

\section{A.3 The Weyl equation}

$$
\begin{array}{rlrl}
\left(\frac{d}{d s}-\Varangle_{1,2}\right) \psi(s) & =0, \\
\psi(\lambda+)-\psi(\lambda-) & =-Q_{+} \chi_{R}, & & \psi(l / 2)=\left(-b_{-}, B_{+}\right) v, \\
\psi(-\lambda+)-\psi(-\lambda-) & =-Q_{-} \chi_{L}, & & \psi(-l / 2)=-\left(B_{-},-b_{+}\right) v .
\end{array}
$$




\section{A.4 Solution of the Weyl equation}

$$
\begin{aligned}
& v=\frac{1}{P}\left(\begin{array}{c}
-\mathrm{e}^{\mathrm{i} \frac{\tau}{4}} B_{-}^{\dagger} \mu_{-} \\
\mathrm{e}^{-\mathrm{i} \frac{\tau}{4}} B_{+}^{\dagger} \mu_{+}
\end{array}\right), \quad\left(\begin{array}{c}
\chi_{R} \\
\chi_{L}
\end{array}\right)=\left(\begin{array}{c}
Q_{+}^{\dagger} \mathrm{e}^{-\lambda \Varangle_{2}} \\
Q_{-}^{\dagger} \mathrm{e}^{\lambda \gtrless_{2}}
\end{array}\right) \Upsilon, \\
& \psi(s)= \begin{cases}\mathrm{e}^{-\mathrm{i} \frac{\tau}{4}} \mathrm{e}^{\left(s-\frac{l}{2}\right) \gtrless_{1}} \mu_{-} & \text {for } \lambda<s<l / 2, \\
\mathrm{e}^{s \gtrless_{2}} \Pi & \text { for }-\lambda<s<\lambda, \\
\mathrm{e}^{\mathrm{i} \frac{\tau}{4}} e^{\left(s+\frac{l}{2}\right) \gtrless_{1}} \mu_{+} & \text {for }-l / 2<s<-\lambda .\end{cases}
\end{aligned}
$$

Here

$$
\begin{aligned}
& 2 g=2\left(y \cosh 2 \lambda z_{2}-\frac{\vec{y} \cdot \vec{z}_{2}}{z_{2}} \sinh 2 \lambda z_{2}\right), \quad \mathcal{P}=\sqrt{\left(T_{1}+t\right)^{2}-z_{1}^{2}} \\
& \mu_{ \pm}=\sqrt{\frac{T_{1}+t+\mathcal{P}}{2}} \pm \sqrt{\frac{T_{1}+t-\mathcal{P}}{2}} \frac{\aleph_{1}}{z_{1}}
\end{aligned}
$$

and

$$
\begin{aligned}
& \Upsilon=\frac{1}{2 g}\left\{\mathrm{e}^{\mathrm{i} \frac{\tau}{4}} \mathrm{e}^{\lambda \gtrless_{2}} \mathrm{e}^{⿱_{1} d} \mu_{+}-\mathrm{e}^{-\mathrm{i} \frac{\tau}{4}} \mathrm{e}^{-\lambda \gtrless_{2}} \mathrm{e}^{-\gtrless_{1} d} \mu_{-}\right\},
\end{aligned}
$$

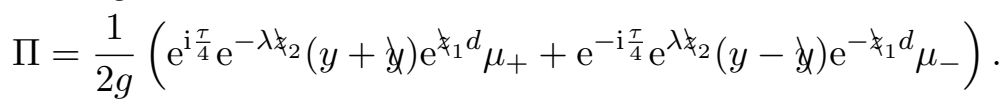

\section{References}

[1] M. F. Atiyah, N. J. Hitchin, V. G. Drinfeld and Yu. I. Manin, Construction of instantons, Phys. Lett. A65 (1978), 185.

[2] W. Nahm, A simple formalism for the BPS monopole, Phys. Lett. B 90 (1980), 413; All self-dual multimonopoles for arbitrary gauge group, CERN-TH.3172 (1981) KEK entry.

[3] W. Nahm, Selfdual monopoles and calorons, BONN-HE-83-16 SPIRES entry Presented at 12th Colloqpium on Group Theoretical Methods in Physics, Trieste, Italy, September 5-10, 1983; Self-dual monopoles and calorons, Physics 201, Springer, New York, 1984, 189-200.

[4] P. B. Kronheimer and H. Nakajima, Yang-Mills instantons on ALE gravitational instantons, Math. Ann. 288(2) (1990), 263-307.

[5] N. Nekrasov and A. S. Schwarz, Instantons on noncommutative $\mathbb{R}^{4}$ and (2,0) superconformal six dimensional theory, Commun. Math. Phys. 198 (1998), 689, arXiv:hep-th/9802068. 
[6] M. R. Douglas and G. W. Moore, D-branes, quivers, and ALE instantons, arXiv:hep-th/9603167; M. R. Douglas, Gauge fields and D-branes, J. Geom. Phys. 28 (1998), 255, arXiv:hep-th/9604198.

[7] C. V. Johnson and R. C. Myers, Aspects of type IIB theory on ALE spaces, Phys. Rev. D55 (1997), 6382, arXiv:hep-th/9610140.

[8] D. E. Diaconescu, D-branes, monopoles and Nahm equations, Nucl. Phys. B503 (1997), 220, arXiv:hep-th/9608163.

[9] T. C. Kraan and P. van Baal, New instanton solutions at finite temperature, Nucl. Phys. A642 (1998), 299, arXiv:hep-th/9805201; Periodic instantons with non-trivial holonomy, Nucl. Phys. B533 (1998), 627, arXiv:hep-th/9805168; Exact T-duality between Calorons and TaubNUT Spaces, Phys. Lett. B428 (1998), 268, arXiv:hep-th/9802049.

[10] K. M. Lee and C. h. Lu, SU(2) calorons and magnetic monopoles, Phys. Rev. D58 (1998), 025011, arXiv:hep-th/9802108.

[11] M. Bianchi, F. Fucito, G. Rossi and M. Martellini, On the ADHM construction on ALE gravitational backgrounds, Phys. Lett. B359 (1995), 49; Explicit construction of Yang-Mills instantons on ALE spaces, Nucl. Phys. B473 (1996), 367, arXiv:hep-th/9601162.

[12] C. N. Pope and A. L. Yuille, A Yang-Mills instanton in Taub-NUT space, Phys. Lett. B78 (1978), 424; A. L. Yuille, Yang-Mills instantons in selfdual space-times, Phys. Lett. B81 (1979), 321.

[13] H. Boutaleb-Joutei, A. Chakrabarti and A. Comtet, Gauge field configurations in curved space-times. 4. Self-dual SU(2) Fields in Multicenter Spaces, Phys. Rev. D21 (1980), 2280.

[14] H. Kim and Y. Yoon, Instanton-meron hybrid in the background of gravitational instantons, Phys. Rev. D63 (2001), 125002, arXiv:hep-th/ 0012055; Effects of gravitational instantons on Yang-Mills Instanton, Phys. Lett. B495 (2000), 169, arXiv: hep-th/0002151.

[15] G. Etesi, Classification of 't Hooft instantons over multi-centered gravitational instantons, Nucl. Phys. B662 (2003), 511, arXiv:hep-th/ 0303146; G. Etesi and T. Hausel, On Yang-Mills instantons over multi-centered gravitational instantons, Commun. Math. Phys. 235 (2003), 275, arXiv:hep-th/0207196; Geometric construction of new Taub-NUT instantons, Phys. Lett. B514 (2001), 189, arXiv: hep-th/0105118.

[16] S. A. Cherkis, Moduli spaces of instantons on the Taub-NUT space, Commun. Math. Phys. 290 (2009), 719, arXiv:0805.1245 [hep-th].

[17] E. Witten, Branes, instantons, and Taub-NUT spaces, JHEP 0906 (2009), 067, arXiv:0902.0948 [hep-th]. 
[18] P. B. Kronheimer, Monopoles and Taub-NUT metrics, MSc. Thesis, Oxford, 1985.

[19] S. A. Cherkis and A. Kapustin, Singular monopoles and supersymmetric gauge theories in three dimensions, Nucl. Phys. B525 (1998), 215, arXiv: hep-th/9711145.

[20] S. A. Cherkis and A. Kapustin, Singular monopoles and gravitational instantons, Commun. Math. Phys. 203 (1999), 713, arXiv:hep-th/ 9803160.

[21] S. A. Cherkis and B. Durcan, Singular Monopoles via the Nahm Transform, JHEP 0804 (2008), 070, arXiv:0712.0850 [hep-th].

[22] S. A. Cherkis and B. Durcan, The 't Hooft-Polyakov monopole in the presence of an 't Hooft operator, Phys. Lett. B671 (2009), 123, arXiv:0711.2318 [hep-th].

[23] G. W. Gibbons and P. Rychenkova, Hyperkaehler quotient construction of BPS Monopole moduli spaces, Commun. Math. Phys. 186 (1997), 585, arXiv: hep-th/9608085.

[24] P. B. Kronheimer, The construction of ALE spaces as hyperkähler quotients, J. Differential Geom. 29 (1989), 665.

[25] J. Hurtubise and M. K. Murray, On the construction of monopoles for the classical groups, Commun. Math. Phys. 122 (1989), 35.

[26] S. K. Donaldson and P. B. Kronheimer, The geomerty of four-manifolds, Oxford University Press, Oxford, 1990.

[27] M. Mamone Capria and S. M. Salamon, Yang-Mills fields on quaternionic spaces, Nonlinearity 1 (1988), 517.

[28] S. A. Cherkis, Instantons on gravitons, arXiv:1007.0044 [hep-th]. 
